Theoret. Appl. Mech., Vol. 30, No. 2, pp. 85-162, Belgrade 2003

\title{
A primer on exterior differential calculus
}

\author{
D.A. Burton*
}

\begin{abstract}
A pedagogical application-oriented introduction to the calculus of exterior differential forms on differential manifolds is presented. Stokes' theorem, the Lie derivative, linear connections and their curvature, torsion and non-metricity are discussed. Numerous examples using differential calculus are given and some detailed comparisons are made with their traditional vector counterparts. In particular, vector calculus on $\mathbb{R}^{3}$ is cast in terms of exterior calculus and the traditional Stokes' and divergence theorems replaced by the more powerful exterior expression of Stokes' theorem. Examples from classical continuum mechanics and spacetime physics are discussed and worked through using the language of exterior forms. The numerous advantages of this calculus, over more traditional machinery, are stressed throughout the article.
\end{abstract}

Keywords: manifolds, differential geometry, exterior calculus, differential forms, tensor calculus, linear connections

\footnotetext{
*Department of Physics, Lancaster University, UK (e-mail: d.burton@lancaster.ac.uk)
} 


\section{Table of notation}

$\begin{array}{ll}\mathcal{M} & \text { a differential manifold } \\ \mathcal{F}(\mathcal{M}) & \text { set of smooth functions on } \mathcal{M} \\ T \mathcal{M} & \text { tangent bundle over } \mathcal{M} \\ T^{*} \mathcal{M} & \text { cotangent bundle over } \mathcal{M} \\ \mathbf{T}_{p}^{q} \mathcal{M} & \text { type }(p, q) \text { tensor bundle over } \mathcal{M} \\ \Lambda_{p} \mathcal{M} & \text { differential } p \text {-form bundle over } \mathcal{M} \\ \Gamma T \mathcal{M} & \text { set of tangent vector fields on } \mathcal{M} \\ \Gamma T^{*} \mathcal{M} & \text { set of cotangent vector fields on } \mathcal{M} \\ \Gamma \mathbf{T}_{p}^{q} \mathcal{M} & \text { set of type }(p, q) \text { tensor fields on } \mathcal{M} \\ \Gamma \Lambda_{p} \mathcal{M} & \text { set of differential } p \text {-forms on } \mathcal{M} \\ \otimes & \text { tensor product } \\ \wedge & \text { exterior product } \\ d & \text { exterior derivative } \\ X & \text { a vector field } \\ \iota_{X} & \text { interior operator with respect to } X \\ \mathcal{L}_{X} & \text { the Lie derivative with respect to } X \\ {[X, Y]} & \text { Lie bracket of vector fields } X \text { and } Y \\ \nabla & \text { a linear connection } \\ \varphi & \text { a diffeomorphism } \\ \varphi_{*} & \text { the push-forward map induced by } \varphi \\ \varphi^{*} & \text { the pull-back map induced by } \varphi \\ \star & \text { Hodge map } \\ \star 1 & \text { an orientation, or volume form } \\ \partial & \text { boundary operator } \\ \{\ldots\} & \text { a set } \\ \left\{X_{1}, X_{2}, \ldots, X_{n}\right\} & \text { a basis for } \Gamma T \mathcal{M} \text { where dim } \mathcal{M}=n \\ \left\{e^{1}, e^{2}, \ldots, e^{n}\right\} & \text { a basis for } \Gamma T^{*} \mathcal{M} \\ \left\{\omega^{1}{ }_{1}, \omega^{1}{ }_{2}, \ldots, \omega^{n}{ }_{n}\right\} & \text { connection } 1 \text {-forms associated with } \nabla \\ \left\{T^{1}, \ldots, T^{n}\right\} & \text { torsion } 2 \text {-forms associated with } \nabla \\ \left\{R^{1}, R_{1}^{1}{ }_{2}, \ldots, R_{n}^{n}\right\} & \text { curvature 2-forms associated with } \nabla \\ & \end{array}$




\section{Contents}

1 Differential manifolds $\quad 89$

2 Tensor fields on manifolds $\quad 90$

2.1 Derivations . . . . . . . . . . . . . . . 90

2.2 Vector fields . . . . . . . . . . . . . . . . . . 93

2.3 Differential 1-forms . . . . . . . . . . . . . . . 95

2.4 Tensor fields of arbitrary degree . . . . . . . . . . . 96

2.4.1 Metric tensor field . . . . . . . . . . . . 98

2.5 Differential forms of arbitrary degree . . . . . . . . . . 99

2.6 Example . . . . . . . . . . . . . . 100

3 The tools of exterior calculus 101

3.1 Example ... . . . . . . . . . . . . . . 104

4 Integration of forms over chains $\quad 106$

4.1 The pull-back of differential forms . . . . . . . . . . . 106

4.2 Cubes and chains . . . . . . . . . . . . . . . 107

4.2.1 Example ................ . . . 108

4.3 Integration and Stokes' theorem . . . . . . . . . . . . 109

4.4 Example . . . . . . . . . . . . . . . . 111

5 Standard vector calculus in terms of exterior calculus 112

5.1 Dot and cross products . . . . . . . . . . . . . 114

5.2 Grad, curl and div . . . . . . . . . . . . . . . 114

5.3 Integral relations . . . . . . . . . . . . . . . . . . . 115

5.4 Applications involving Stokes' theorem on $\mathbb{R}^{3}$. . . . 118

6 Differential operators on tensor bundles 122

6.1 The push-forward map . . . . . . . . . . . . . . 123

6.2 One-parameter families of diffeomorphisms . . . . . . . 124

6.2.1 Example . . . . . . . . . . . . 126 
6.3 The Lie derivative . . . . . . . . . . . . . . . . . . . . . . 127

6.3 .1 Example . . . . . . . . . . . . . . . . . . . . . . 129

6.4 Linear connections on tensor bundles . . . . . . . . . 130

6.4 .1 Example . . . . . . . . . . . . . . . . . . . . . . . 131

6.4.2 Connection 1-forms . . . . . . . . . . . . . . . 132

6.4 .3 Torsion . . . . . . . . . . . . . . . . . . 133

6.4.4 Curvature . . . . . . . . . . . . . . . . . . . . . 134

6.4.5 The Bianchi identities . . . . . . . . . . . 135

6.4.6 Non-metricity . . . . . . . . . . . . . . . 136

6.4.7 Covariant exterior derivatives . . . . . . . . 137

6.4.8 Covariant derivatives, parallel transport and autoparallels . . . . . . . . . . . . . . 138

6.4.9 The Levi-Civita connection . . . . . . . . . 140

6.4.10 Example : differential geometry on the 2-sphere 142

7 Newtonian continuum mechanics 144

7.1 Example : Hydrodynamics of perfect fluids . . . . . . 148

8 Differential forms on spacetime 151

8.1 Electromagnetism . . . . . . . . . . . . . . . . . . . 152

8.2 Einstein's equations . . . . . . . . . . . . . . . . 154

8.2.1 Conservation laws induced by stress-energy tensors . . . . . . . . . . . . 156

8.2 .2 Example : Dust . . . . . . . . . . . . . . 157

8.2 .3 Common stress forms . . . . . . . . . . . 158

\section{Introduction}

Differential geometry is a powerful mathematical tool and pervades many branches of physics. Physical theories are often naturally and concisely expressed in terms of differential geometric concepts. The 
aim of this article is to give an application-oriented pedagogical introduction to some of the ideas in differential geometry, specifically the notion of exterior differential forms, and to explicitly demonstrate the power of the formalism. It is shown how the calculus of differential forms gives rise to a concise alternative to traditional vector and tensor calculus and the corresponding treatments of field theories. Numerous examples are discussed and include applications in classical continuum mechanics and relativistic spacetime physics.

Traditional Gibbs vectors and matrices will be distinguished from differential geometric vector fields by using a bold face font. For example $\boldsymbol{v}$ is a conventional vector field, whilst $V$ is a differential geometric vector field. A function on an open subset $\mathcal{U} \subset \mathbb{R}^{m}$ into $\mathbb{R}^{n}$ is said to be smooth if its partial derivatives to all orders exist and are continuous.

\section{Differential manifolds}

Loosely speaking, differential manifolds are generalizations of the concept of Euclidean spaces. Any point in a differential manifold has an open neighbourhood that can be smoothly mapped onto an open subset of a Euclidean space. Unlike Euclidean spaces, arbitrary differential manifolds require more than one open set to cover them.

Let $\mathcal{M}$ be a set. A pair $(\mathcal{U}, \phi)$, where $\mathcal{U} \subseteq \mathcal{M}$ and $\phi: \mathcal{U} \rightarrow \mathbb{R}^{n}$ is a one-to-one map onto an open set $\phi(\mathcal{U}) \subseteq \mathbb{R}^{n}$, is called a chart on $\mathcal{M}$. Two charts $(\mathcal{U}, \phi)$ and $(\mathcal{V}, \psi)$ are called compatible if either $\mathcal{U} \cap \mathcal{V}=\varnothing$ or else $\mathcal{U} \cap \mathcal{V} \neq \varnothing, \phi(\mathcal{U} \cap \mathcal{V})$ and $\psi(\mathcal{U} \cap \mathcal{V})$ are open in $\mathbb{R}^{n}$ and $\phi \circ \psi^{-1}: \psi(\mathcal{U} \cap \mathcal{V}) \rightarrow \phi(\mathcal{U} \cap \mathcal{V})$ is smooth with a smooth inverse (see figure 1). An atlas is a family of charts, any two of which are compatible and whose domains cover $\mathcal{M}$. Two atlases are called equivalent if their union is an atlas, and a set $\mathcal{M}$ with an equivalence class of such atlases is called a differential manifold (or, simply a man- 
ifold $\left.{ }^{1}\right)$. The dimension of a manifold is the dimension of the range of all of the chart maps in some (and, hence, any equivalent) atlas. Let $\mathcal{M}$ be a manifold with dimension $m$ and $\mathcal{N}$ be a manifold with dimension $n$. A function $f$ on $\mathcal{M}$ into $\mathcal{N}$ is said to be smooth if for every $p \in \mathcal{U}$ there is a chart $(\mathcal{U}, \phi)$ for $\mathcal{M}$ and a chart $(\mathcal{V}, \psi)$ for $\mathcal{N}$ at $f(p)$ with $f(\mathcal{U}) \subseteq \mathcal{V}$ such that the partial derivatives of

$$
\psi \circ f \circ \phi^{-1}: \phi(\mathcal{U}) \subseteq \mathbb{R}^{m} \longrightarrow \psi(\mathcal{V}) \subseteq \mathbb{R}^{n}
$$

exist and are continuous to all orders, i.e. $\psi \circ f \circ \phi^{-1}$ is smooth.

\section{Tensor fields on manifolds}

\subsection{Derivations}

Let $\mathcal{F}(\mathcal{M})$ be the set of smooth functions on an $n$-dimensional manifold $\mathcal{M}$ into $\mathbb{R}$. A derivation on the algebra of $\mathcal{F}(\mathcal{M})$ is a map $X$ such that

$$
\begin{aligned}
& X: \mathcal{F}(\mathcal{M}) \rightarrow \mathcal{F}(\mathcal{M}), \\
& X(\lambda f+\mu h)=\lambda V f+\mu V h, \\
& X(f h)=X f h+f X h,
\end{aligned}
$$

where $\lambda, \mu \in \mathbb{R}, f, h \in \mathcal{F}(\mathcal{M})$ and where the shorthand $X f \equiv X(f)$ has been used. The expression of $X f \in \mathcal{F}(\mathcal{M})$ with respect to the $\operatorname{chart}(\mathcal{U}, \phi), x^{a}=\phi^{a}(p), a=1, \ldots, n, p \in \mathcal{U}$ on $\mathcal{M}$ is $^{2}$

$$
X f=\xi^{a} \frac{\partial\left(f \circ \phi^{-1}\right)}{\partial x^{a}} \circ \phi
$$

\footnotetext{
${ }^{1}$ The standard usage of the term manifold is reserved for objects that have less structure than differential manifolds. However, in this article we use manifold as an abbreviation for differential manifold.

${ }^{2}$ The Einstein summation convention is adhered to throughout this document, i.e. repeated labels are summed over their ranges.
} 


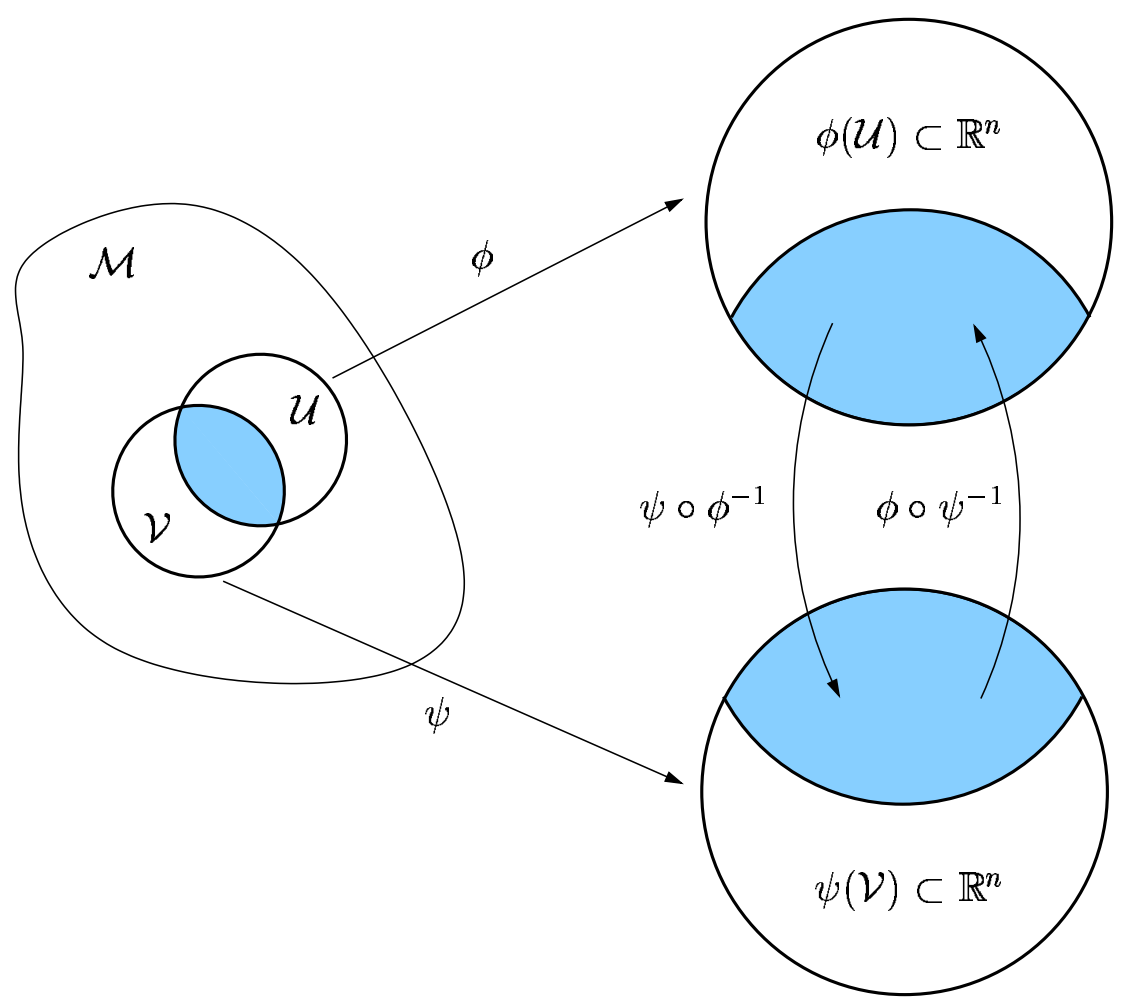

Figure 1: Loosely speaking, a differential manifold $\mathcal{M}$ is a collection of points whose open neighbourhoods can be smoothly mapped onto open subsets of a Euclidean space. All of the maps shown in this figure are smooth with smooth inverses. The dimension of $\mathcal{M}$ is $n$. 
where $\left\{\xi^{a}\right\}, \xi^{a}: \mathcal{U} \rightarrow \mathbb{R}^{n}$, are known as the components of $X$ with respect to $(\mathcal{U}, \phi)$. Similarly, with respect to another chart $(\mathcal{V}, \psi)$, $x^{\prime a}=\psi^{a}(p), p \in \mathcal{V}$

$$
X f=\xi^{\prime a} \frac{\partial\left(f \circ \psi^{-1}\right)}{\partial x^{a}} \circ \psi
$$

on $\mathcal{U} \cap \mathcal{V}$. The components $\left\{\xi^{a}\right\}$ and $\left\{\xi^{\prime a}\right\}$ of $X$ are related by applying the chain rule to (2). It can be shown that

$$
X f=\xi^{\prime a} \Phi_{\mathcal{U} \mathcal{V}_{a}^{b}} \frac{\partial\left(f \circ \phi^{-1}\right)}{\partial x^{b}} \circ \phi
$$

where the transition function $\Phi_{\mathcal{U V}}$ is

$$
\begin{aligned}
& \Phi_{\mathcal{U V}}: \mathcal{U} \cap \mathcal{V} \rightarrow \mathbb{R}^{n}, \\
& \Phi_{\mathcal{U V}{ }_{b}}=\frac{\partial\left(\phi \circ \psi^{-1}\right)^{a}}{\partial x^{\prime b}} \circ \psi
\end{aligned}
$$

where for $p \in \mathcal{U} \cap \mathcal{V}$ with $x^{a}=\phi^{a}(p), x^{a}=\psi^{a}(p)$,

$$
x^{a}=\left(\phi \circ \psi^{-1}\right)^{a}\left(x^{1}, \ldots x^{\prime n}\right) .
$$

Comparing (3) and (1) we note that

$$
\xi^{a}=\Phi_{\mathcal{U} \mathcal{V}_{b}^{a}} \xi^{\prime b}
$$

or, equivalently,

$$
\xi^{\prime a}=\Phi_{\mathcal{V U}}^{a} \xi^{b} .
$$

since

$$
\Phi_{\mathcal{U V}}^{a} \Phi_{\mathcal{V U}_{c}^{b}}^{b}=\delta_{c}^{a}
$$




\section{$2.2 \quad$ Vector fields}

Each point $p \in \mathcal{M}$ is equipped with an $n$-dimensional vector space $T_{p} \mathcal{M}$, called the tangent space at $p$. Elements of $T_{p} \mathcal{M}$ are called tangent vectors at $p$. The tangent spaces are collected together to form a $2 n$-dimensional manifold $T \mathcal{M}$,

$$
T \mathcal{M}=\bigcup_{p \in \mathcal{M}} T_{p} \mathcal{M}
$$

known as the tangent bundle of $\mathcal{M}$, which is an example of a fibre bundle [9]. Crudely speaking, a section of a fibre bundle, such as $T \mathcal{M}$, is an assignment of a point (in this case a tangent vector at $p$ ) in each fibre (in this case $T_{p} \mathcal{M}$ ) to its base point in the base manifold (in this case $p \in \mathcal{M}$ ) that varies smoothly over the base manifold (see figure 2). Elements of the space of sections of $T \mathcal{M}$, denoted $\Gamma T \mathcal{M}$, are called vector fields. Expressed with respect to the chart $(\mathcal{U}, \phi)$, $x^{a}=\phi^{a}(p), p \in \mathcal{U}$ a vector field $X \in \Gamma T \mathcal{M}$ is written

$$
X=\xi^{a} \frac{\partial}{\partial x^{a}}
$$

where $\xi^{a}: \mathcal{U} \rightarrow \mathbb{R}^{n}$ are the components of $X$ with respect to $(\mathcal{U}, \phi)$. At each point $p \in \mathcal{U}$ the set $\left\{\partial / \partial x^{a}\right\}$ is a vector basis for $T_{p} \mathcal{M}$. This notation reflects the fact that the derivations on the algebra of $\mathcal{F}(\mathcal{M})$ and the vector fields on $\mathcal{M}$ are in one-to-one correspondence. With respect to $(\mathcal{V}, \psi), x^{a}=\psi^{a}(p), p \in \mathcal{V}$

$$
X=\xi^{\prime a} \frac{\partial}{\partial x^{\prime a}}
$$

and so applying (6) to (7) and (8) we obtain

$$
\frac{\partial}{\partial x^{\prime a}}=\Phi_{\mathcal{U}} \mathcal{V}^{b} \frac{\partial}{\partial x^{b}} .
$$

on $\mathcal{U} \cap \mathcal{V}$ 


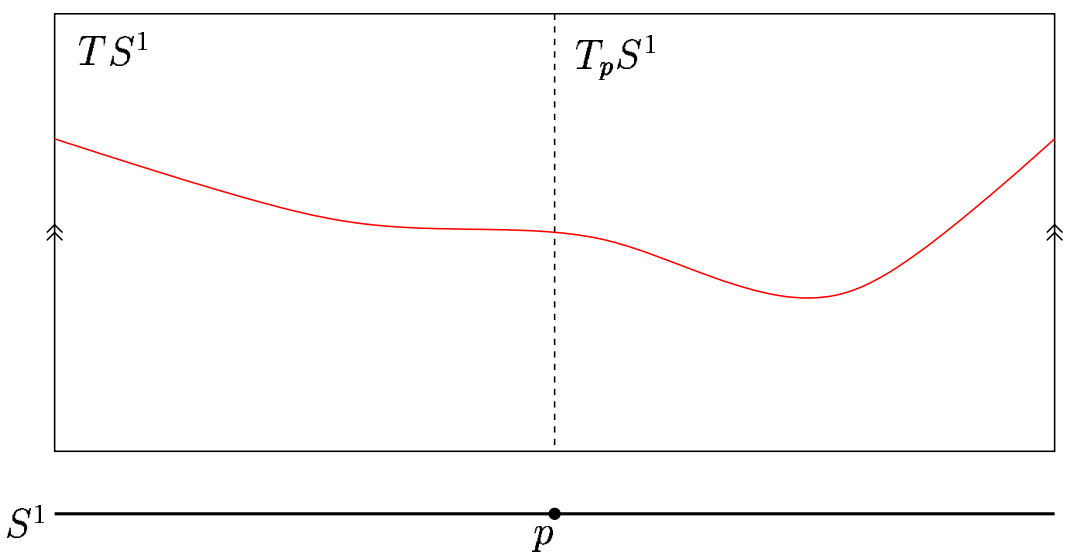

Figure 2: This figure illustrates the idea of a fibre bundle (specifically, the tangent bundle $T S^{1}$ on the circle $S^{1}$ ). The tangent space $T_{p} S^{1}$ (a fibre of $T S^{1}$ ) at $p \in S^{1}$ is shown by the dotted line. The union of $T_{p} S^{1}$ for all $p$ yields the fibre bundle $T S^{1}$ and the arrows show how the edges should be identified, i.e. $T S^{1}=S^{1} \times \mathbb{R}$. The curve is a section of $T S^{1}$ i.e. a vector field on $S^{1}$. Not all tangent bundles are product bundles. For example $T S^{2} \neq S^{2} \times \mathbb{R}^{2}$ because all vector fields on $S^{2}$ must vanish somewhere. 


\subsection{Differential 1-forms}

A 1 -form $\alpha_{p}$ at $p$ is a linear map from $T_{p} \mathcal{M}$ to $\mathbb{R}$, i.e. $\alpha_{p}$ is an element of the dual space $T_{p}^{*} \mathcal{M}$. The space $T^{*} \mathcal{M}$

$$
T^{*} \mathcal{M}=\bigcup_{p \in \mathcal{M}} T_{p}^{*} \mathcal{M}
$$

is known as the cotangent bundle of $\mathcal{M}$. Differential 1-forms are elements of the space of sections of $T^{*} \mathcal{M}$, denoted $\Gamma T^{*} \mathcal{M}$, and they are linear maps on vector fields into $\mathcal{F}(\mathcal{M})$. Thus,

$$
\begin{aligned}
& \alpha(X) \in \mathcal{F}(\mathcal{M}), \\
& \alpha(f X) \equiv f \alpha(X), \\
& \alpha(X+Y) \equiv \alpha(X)+\alpha(Y), \\
& (\alpha+\beta)(X) \equiv \alpha(X)+\beta(X),
\end{aligned}
$$

where $f \in \mathcal{F}(\mathcal{M}), X \in \Gamma T \mathcal{M}, Y \in \Gamma T \mathcal{M}, \alpha \in \Gamma T^{*} \mathcal{M}$ and $\beta \in$ $\Gamma T^{*} \mathcal{M}$. We can consider vector fields as linear maps on differential 1-forms by defining

$$
X(\alpha) \equiv \alpha(X)
$$

thus identifying $T^{* *} \mathcal{M}$ with $T \mathcal{M}$. The expressions for $\alpha$ with respect to the charts $(\mathcal{U}, \phi)$ and $(\mathcal{V}, \psi)$ used earlier are

$$
\begin{aligned}
\alpha & =\alpha_{a} d x^{a} \\
& =\alpha_{a}^{\prime} d x^{\prime a} .
\end{aligned}
$$

where $\left\{d x^{\prime a}\right\}$ and $\left\{d x^{a}\right\}$ are bases for $\Gamma T^{*} \mathcal{M}$ valid on $\mathcal{V}$ and $\mathcal{U}$ respectively. The bases $\left\{\partial / \partial x^{a}\right\}$ and $\left\{d x^{a}\right\}$ are $d u a l$,

$$
d x^{a}\left(\partial / \partial x^{b}\right) \equiv \delta_{b}^{a}
$$


as are $\left\{\partial / \partial x^{\prime a}\right\}$ and $\left\{d x^{\prime a}\right\}$

$$
d x^{\prime a}\left(\partial / \partial x^{\prime b}\right) \equiv \delta_{b}^{a}
$$

where $\delta_{b}^{a}=1$ if $a=b$ and $\delta_{b}^{a}=0$ if $a \neq b\left(\delta_{b}^{a}\right.$ is the Kronecker delta $)$. The contraction $\alpha(X)$ is chart-independent so using (15) and (6)

$$
\begin{aligned}
\alpha(X) & =\alpha_{a}^{\prime} \xi^{\prime b} d x^{a}\left(\frac{\partial}{\partial x^{\prime b}}\right) \\
& =\alpha_{a}^{\prime} \xi^{\prime a} \\
& =\alpha_{a}^{\prime} \Phi_{\mathcal{V U}}^{a} \xi^{b} . \\
& =\alpha_{a} \xi^{a}
\end{aligned}
$$

where the last line is expressed with respect to $(\mathcal{U}, \phi)$ and so

$$
\alpha_{a}^{\prime}=\Phi_{\mathcal{U} \mathcal{V}_{a}^{b}}^{b} \alpha_{b}
$$

Thus, using (14), the differential 1-form bases are related by

$$
d x^{\prime a}=\Phi_{\mathcal{V U}}{ }_{b}^{a} d x^{b} .
$$

on $\mathcal{U} \cap \mathcal{V}$.

\subsection{Tensor fields of arbitrary degree}

Elements of the vector spaces $\Gamma T \mathcal{M}$ and $\Gamma T^{*} \mathcal{M}$ are used to construct multilinear mappings into $\mathcal{F}(\mathcal{M})$. The space $\mathbf{T}_{r p}^{s} \mathcal{M}$ at $p \in \mathcal{M}$ consists of all multilinear mappings on the product of the $r$ th-order product of $T_{p} \mathcal{M}$ and the $s$ th-order product of $T_{p}^{*} \mathcal{M}$. Since $T_{p}^{*} \mathcal{M}$ is the space of linear maps on $T_{p} \mathcal{M}$ and $T_{p}^{* *} \mathcal{M}=T_{p} \mathcal{M}$ is the space of linear maps on $T_{p}^{*} \mathcal{M}$ we see that

$$
\mathbf{T}_{r p}^{s} \mathcal{M}=\underbrace{\left(T_{p}^{*} \mathcal{M} \times T_{p}^{*} \mathcal{M} \times \ldots T_{p}^{*} \mathcal{M}\right)}_{r \text { times }} \times \underbrace{\left(T_{p} \mathcal{M} \times T_{p} \mathcal{M} \times \ldots T_{p} \mathcal{M}\right)}_{s \text { times }} .
$$


A smooth type $(r, s)$ tensor field $T$ is an element of the space of sections of the type $(r, s)$ tensor bundle

$$
\mathbf{T}_{r}^{s} \mathcal{M}=\bigcup_{p \in \mathcal{M}} \mathbf{T}_{r p}^{s} \mathcal{M}
$$

i.e. $T \in \Gamma \mathbf{T}_{r}^{s} \mathcal{M}$. The integer $r$ is called the covariant degree of $T$ whilst $s$ is its contravariant degree. Special examples of tensor bundles are the tangent bundle $T \mathcal{M}=\mathbf{T}_{0}^{1} \mathcal{M}$ and the cotangent bundle $T^{*} \mathcal{M}=\mathbf{T}_{1}^{0} \mathcal{M}$.

The tensor product $\otimes$ has the properties

$$
\begin{aligned}
& (\alpha \otimes T)\left(X, Y_{1}, \ldots Y_{r}, \alpha_{1}, \ldots, \alpha_{s}\right) \equiv \alpha(X) T\left(Y_{1}, \ldots Y_{r}, \alpha_{1}, \ldots, \alpha_{s}\right), \\
& (\alpha \otimes \beta)(X, Y) \equiv \alpha(X) \beta(Y)
\end{aligned}
$$

with

$$
X(\alpha) \equiv \alpha(X)
$$

where $X, Y, Y_{1}, \ldots, Y_{r} \in \Gamma T \mathcal{M}$ and $\alpha, \alpha_{1}, \ldots, \alpha_{s}, \beta \in \Gamma T^{*} \mathcal{M}$. The linearity properties of the tensor product are induced from (11), (12) and (13). For example

$$
\begin{aligned}
(\alpha \otimes \beta)(f X, Y) & =f \alpha(X) \beta(Y) \\
& =(\alpha \otimes \beta)(X, f Y) \\
& =(f \alpha \otimes \beta)(X, Y) \\
& =(\alpha \otimes f \beta)(X, Y)
\end{aligned}
$$

where $f \in \mathcal{F}(\mathcal{M})$. With respect to the chart $(\mathcal{U}, \phi)$ the tensor $T$ is

$$
T=T_{a_{1} \ldots a_{r}}^{b_{1} \ldots b_{s}} d x^{a_{1}} \otimes d x^{a_{2}} \otimes \ldots \otimes d x^{a_{r}} \otimes \frac{\partial}{\partial x^{b_{1}}} \otimes \frac{\partial}{\partial x^{b_{2}}} \otimes \ldots \otimes \frac{\partial}{\partial x^{b_{s}}} .
$$




\subsubsection{Metric tensor field}

A metric tensor on $\mathcal{M}$ is a type $(2,0)$ symmetric non-degenerate tensor field $g \in \Gamma \mathbf{T}_{2}^{0} \mathcal{M}$. An orthonormal co-frame $\left\{e^{a}\right\}$ is a set of $n=\operatorname{dim} \mathcal{M}$ linearly independent sections of $T^{*} \mathcal{M}$ with respect to which the metric has the form

$$
g=\eta_{a b} e^{a} \otimes e^{b}
$$

where $\eta_{a b}= \pm 1$ if $a=b$ and $\eta_{a b}=0$ if $a \neq b$. If $\eta_{a b}=+1$ when $a=b$ then $\mathcal{M}$ is said to be Riemannian. Otherwise $\mathcal{M}$ is called semi-Riemannian or, alternatively, pseudo-Riemannian. A Lorentzian manifold $\mathcal{M}$ is semi-Riemannian with $\eta_{a b}=\operatorname{diag}(-1,1, \ldots, 1) .{ }^{3}$ The metric tensor possesses an inverse $g^{-1}$ which is the type $(0,2)$ tensor field

$$
g^{-1}=\eta^{a b} X_{a} \otimes X_{b}
$$

where $\left\{X_{a}\right\}$ is dual to $\left\{e^{a}\right\}$, i.e.

$$
e^{a}\left(X_{b}\right)=\delta_{b}^{a}
$$

and where

$$
\eta^{a b} \eta_{b c}=\delta_{c}^{a}
$$

The frame $\left\{X_{a}\right\}$ (as well as the co-frame $\left\{e^{a}\right\}$ ) is said to be orthonormal.

The metric establishes an isomorphism between $T \mathcal{M}$ and $T^{*} \mathcal{M}$. Given any $X \in \Gamma T \mathcal{M}$ we can construct the differential 1-form $g(X,-) \equiv$ $g\left(X, X_{a}\right) e^{a}$. Conversely, given any differential 1 -form $\alpha$ we have the

\footnotetext{
${ }^{3}$ For example, $\eta_{a b}=\operatorname{diag}(-1,1,1,1)$ if $\mathcal{M}$ is a spacetime.
} 
vector field $g^{-1}(\alpha,-) \equiv g^{-1}\left(\alpha, e^{a}\right) X_{a}$. For convenience we use the notation

$$
\begin{aligned}
& \tilde{X} \equiv g(X,-), \\
& \tilde{\alpha} \equiv g^{-1}(\alpha,-) .
\end{aligned}
$$

Thus $\tilde{\tilde{X}}=X$ and $\tilde{\tilde{\alpha}}=\alpha$.

\subsection{Differential forms of arbitrary degree}

The totally antisymmetric type $(r, 0)$ tensor fields on $\mathcal{M}$ are sections of the rth exterior bundle $\Lambda_{r} \mathcal{M} \subset \mathbf{T}_{r}^{0} \mathcal{M}$ and are known as differential forms of degree $r$ or differential $r$-forms. The bundle of differential 0 -forms $\Lambda_{0} \mathcal{M}$ is defined so that $\Gamma \Lambda_{0} \mathcal{M}=\mathcal{F}(\mathcal{M})$ i.e. differential 0forms are scalar functions on $\mathcal{M}$. Note that if $\mathcal{M}$ is an $n$-dimensional manifold then a differential $r$-form, with respect to an arbitrary chart, has $n ! /(r !(n-r) !)$ components. In other words, the vector space of differential $r$-forms on an $n$-dimensional manifold has dimension $n ! /(r !(n-r) !)$. Let $\alpha$ be a differential $r$-form and $\beta$ be a differential $s$ form. The exterior product of $\alpha$ and $\beta$, denoted $\alpha \wedge \beta$, is the differential $(r+s)$-form given by

$$
\alpha \wedge \beta \equiv \operatorname{Alt}(\alpha \otimes \beta)
$$

where $\operatorname{Alt}(T)$ is the totally antisymmetric part of the type $(r, 0)$ tensor $T$. For example, if $\alpha$ and $\beta$ are both differential 1 -forms

$$
\alpha \wedge \beta=\frac{1}{2}(\alpha \otimes \beta-\beta \otimes \alpha)
$$

It can be shown that for $\alpha \in \Gamma \Lambda_{r} \mathcal{M}$ and $\beta \in \Gamma \Lambda_{s} \mathcal{M}$

$$
\alpha \wedge \beta=(-1)^{r s} \beta \wedge \alpha
$$


and that the exterior product is associative

$$
\begin{aligned}
\alpha \wedge(\beta \wedge \gamma) & =(\alpha \wedge \beta) \wedge \gamma \\
& \equiv \alpha \wedge \beta \wedge \gamma
\end{aligned}
$$

where $\gamma \in \Gamma \Lambda_{t} \mathcal{M}$. Conventionally, the exterior product symbol is dropped when applied to a differential 0 -form $f$ and a differential $r$ form $\alpha$, i.e. $f \alpha \equiv f \wedge \alpha=\alpha \wedge f$.

All differential $r$-forms are special examples of sections of the exterior bundle $\Lambda \mathcal{M}$. A general section of $\Lambda \mathcal{M}$ consists of linear combinations of differential forms of different degrees. Such differential forms are termed inhomogenous, whilst differential $r$-forms are called homogenous. The vector space of inhomogenous differential forms on an $n$-dimensional manifold has dimension $2^{n}$.

\subsection{Example}

Let $V$ be a vector field and $\alpha$ be a differential 1-form on the 2dimensional manifold $\mathcal{M}=\mathbb{R}^{2}$. Let $(x, y)=\phi(p)$ be the components of a Cartesian chart $(\mathcal{U}, \phi)$ at $p \in \mathcal{U}=\mathbb{R}^{2}$. This means that the metric tensor has the form

$$
g=d x \otimes d x+d y \otimes d y
$$

over $\mathcal{U}$. If $(r, \theta)=\psi(p)$ are the components of the polar chart $(\mathcal{V}, \psi)$ at $p \in \mathcal{V}=\mathbb{R}^{2}-\{0\}$ given by

$$
(x, y)=(r \cos \theta, r \sin \theta)
$$

and since

$$
\begin{aligned}
(x, y) & =\phi(p) \\
& =\phi \circ \psi^{-1} \circ \psi(p) \\
& =\phi \circ \psi^{-1}(r, \theta)
\end{aligned}
$$


we see that

$$
\phi \circ \psi^{-1}(r, \theta)=(r \cos \theta, r \sin \theta) .
$$

Thus, using (17) and (5) we find that

$$
\begin{aligned}
& d x=\cos \theta d r-r \sin \theta d \theta, \\
& d y=\sin \theta d r+r \cos \theta d \theta
\end{aligned}
$$

and so

$$
g=d r \otimes d r+r^{2} d \theta \otimes d \theta .
$$

Note that $\{\partial / \partial r,(1 / r) \partial / \partial \theta\}$ and $\{d r, r d \theta\}$ are a dual orthonormal frame and co-frame valid over $\mathcal{V}$.

An inhomogenous form $\Phi$ on $\mathcal{M}$ expressed with respect to $(\mathcal{U}, \phi)$ has the structure

$$
\Phi=a(x, y)+b(x, y) d x+c(x, y) d y+f(x, y) d x \wedge d y .
$$

\section{The tools of exterior calculus}

Let $\alpha$ be a differential 1-form, $\beta$ be a differential $p$-form, $\gamma$ be an arbitrary degree differential form, $f$ be a scalar field and $X$ be a vector field on an $n$-dimensional manifold $\mathcal{M}$. The exterior derivative $d$ on differential forms is defined by the properties

$$
\begin{aligned}
& d f(X)=X f \\
& d(\beta \wedge \gamma)=d \beta \wedge \gamma+(-1)^{p} \beta \wedge d \gamma, \\
& d d \gamma=0 .
\end{aligned}
$$

With respect to the chart $(\mathcal{U}, \phi)$

$$
X=\xi^{a} \frac{\partial}{\partial x^{a}}
$$


and so, referring to (1)

$$
X f=\xi^{a} \frac{\partial}{\partial x^{a}}\left(f \circ \phi^{-1}\right) \circ \phi .
$$

However,

$$
d f(X)=\xi^{a} d f\left(\frac{\partial}{\partial x^{a}}\right)
$$

and so, since $X$ is arbitrary, the exterior derivative on 0 -forms has the local form

$$
d f=\left[\frac{\partial}{\partial x^{a}}\left(f \circ \phi^{-1}\right) \circ \phi\right] d x^{a} .
$$

The interior operator $\iota_{X}$ with respect to the vector field $X$ is defined by

$$
\begin{aligned}
& \iota_{X} \alpha=\alpha(X), \\
& \iota_{X}(\beta \wedge \gamma)=\iota_{X} \beta \wedge \gamma+(-1)^{p} \beta \wedge \iota_{X} \gamma, \\
& \iota_{X} \iota_{X} \gamma=0 .
\end{aligned}
$$

Both $d$ and $\iota_{X}$ are extended to inhomogenous differential forms by linearity. Specifically, if

$$
\alpha=\sum_{q=0}^{n} \alpha_{q}
$$

where $\alpha_{q} \in \Gamma \Lambda_{q} \mathcal{M}$ then

$$
\begin{aligned}
& d \alpha=\sum_{q=0}^{n} d \alpha_{q}, \\
& \iota_{X} \alpha=\sum_{q=0}^{n} \iota_{X} \alpha_{q} .
\end{aligned}
$$


The dimension of the vector space of differential $p$-forms is $n ! /(p !(n-$ $p) !)$. Thus, the vector spaces of differential $p$-forms and $(n-p)$-forms have the same dimension. The Hodge map $\star$ is a linear isomorphism between the vector spaces of differential $p$ - and $(n-p)$-forms satisfying

$$
\begin{aligned}
& \star(\gamma \wedge \tilde{X})=\iota_{X} \star \gamma, \\
& \star(f \gamma)=f \star \gamma
\end{aligned}
$$

and is completely defined by specifying an orientation, or volume form, denoted $\star 1 \in \Gamma \Lambda_{n} \mathcal{M}$. The orientation is specified through the metric tensor $g$ on $\mathcal{M}$ and has the form

$$
\star 1= \pm e^{1} \wedge e^{2} \wedge \cdots \wedge e^{n}
$$

where $\left\{e^{a}\right\}$ is any orthonormal co-frame (see subsection 2.4.1). The choice of sign is a matter of taste, and on $\mathbb{R}^{3}$ can be identified with the choice of left- or right-handedness of orthonormal frames. Using (28) and (27) a repeated application of the Hodge map on a $p$-form $\beta$ can be shown to yield

$$
\star \star \beta=\operatorname{det}(\boldsymbol{\eta})(-1)^{p(n-p)} \beta
$$

where $\boldsymbol{\eta}$ is the matrix of components $\eta_{a b}=g\left(X_{a}, X_{b}\right)$ of the metric $g$ with respect to an orthonormal frame $\left\{X_{a}\right\}$ and $\beta \in \Gamma \Lambda_{p} \mathcal{M}$. Thus, the inverse Hodge map ${ }^{-1}$ is

$$
{ }^{-1} \beta=\operatorname{det}(\boldsymbol{\eta})(-1)^{p(n-p)} \star \beta .
$$

A useful identity involving the interior operator and Hodge map is that

$$
\tilde{X} \wedge \star \beta=-(-1)^{p} \star \iota_{X} \beta
$$


where, again, $\beta \in \Gamma \Lambda_{p} \mathcal{M}$. Therefore, the metric contraction of two differential 1-forms $\alpha$ and $\beta$ on $\mathcal{M}$ can be expressed in the form

$$
g^{-1}(\alpha, \beta)={ }_{\star}^{-1}(\alpha \wedge \star \beta) .
$$

Indeed, the Hodge map is used to define an inner product on homogenous differential forms on Riemannian manifolds and an indefinite inner product on semi-Riemannian manifolds :

$$
\alpha \cdot \beta \equiv{ }_{\star}^{-1}(\alpha \wedge \star \beta)
$$

where $\alpha$ and $\beta$ are homogenous differential forms with the same degree. A differential form $\alpha$ that satisfies

$$
d \alpha=0
$$

is said to be closed. If a differential form $\beta$ can be written

$$
\beta=d \gamma
$$

where $\gamma$ is another differential form then $\beta$ is called exact. A beautiful, and very powerful, lemma due to Poincaré is that any closed differential form can be written locally as an exact differential form. More precisely, if $d \alpha=0$ on $\mathcal{M}$ then for any $p \in \mathcal{M}$ there exists an open neighbourhood of $p$ on which $\alpha=d \beta$. That this cannot, in general, be done globally is a consequence of the topology of $\mathcal{M}$.

\subsection{Example}

Let $\alpha$ be a 1 -form on a 2 -dimensional differential manifold $(\mathcal{M}, g)$. With respect to a chart $(\mathcal{U}, \phi) \alpha$ has the form

$$
\alpha=a(x, y) d x+b(x, y) d y
$$


Note that

$$
\begin{aligned}
d \alpha & =d a \wedge d x+a d(d x)+d b \wedge d y+b d(d y), \\
& =d a \wedge d x+d b \wedge d y
\end{aligned}
$$

using (22) and (23). Since $\{d x, d y\}$ is dual to $\{\partial / \partial x, \partial / \partial y\}$ we note that

$$
d a=d a(\partial / \partial x) d x+d a(\partial / \partial y) d y
$$

and so, by (21)

$$
d a=\frac{\partial a}{\partial x} d x+\frac{\partial a}{\partial y} d y .
$$

Using the symmetry properties of the exterior product

$$
\begin{aligned}
& d x \wedge d x=d y \wedge d y=0 \\
& d y \wedge d x=-d x \wedge d y
\end{aligned}
$$

and we conclude that

$$
d \alpha=\left(\frac{\partial b}{\partial x}-\frac{\partial a}{\partial y}\right) d x \wedge d y .
$$

With respect to $(\mathcal{U}, \phi)$ the volume form $\star 1$ will be

$$
\star 1=h d x \wedge d y
$$

where $h$ is the component of $\star 1$ with respect to $(\mathcal{U}, \phi)$. The Hodge dual of $\alpha$ is

$$
\begin{aligned}
\star \alpha & =\iota_{\tilde{\alpha}} \star 1 \\
& =\iota_{\tilde{\alpha}}(h d x \wedge d y) \\
& =h \iota_{\tilde{\alpha}} d x \wedge d y-h d x \wedge \iota_{\tilde{\alpha}} d y \\
& =h g^{-1}(\alpha, d x) d y-h g^{-1}(\alpha, d y) d x
\end{aligned}
$$

using (20), (27), (25) and (24). 


\section{Integration of forms over chains}

\subsection{The pull-back of differential forms}

A smooth map $f: \mathcal{M} \rightarrow \mathcal{N}$ induces the pull-back map $f^{*}: \Lambda \mathcal{N} \rightarrow$ $\Lambda \mathcal{M}$ that takes differential forms on $\mathcal{N}$ to differential forms on $\mathcal{M}$. Let $(\mathcal{U}, \phi)$ be a chart on $\mathcal{M}$ with components $x^{a}=\phi^{a}(p)$ at $p \in \mathcal{M}$ and let $(\mathcal{V}, \psi)$ be a chart on $\mathcal{N}$ with components $y^{\mu}=\psi^{\mu}(q)$ at $q \in \mathcal{N}$. The pull-back $f^{*} h$ of the 0 -form $h \in \Gamma \Lambda_{0} \mathcal{N}=\mathcal{F}(\mathcal{M})$ with respect to $f$ is

$$
f^{*} h \equiv h \circ f
$$

The pull-back $f^{*} \alpha$ of $\alpha \in \Gamma \Lambda_{1} \mathcal{N}$, where

$$
\alpha=\alpha_{\mu} d y^{\mu}
$$

with respect to $(\mathcal{V}, \psi)$, is the differential 1 -form on $\mathcal{M}$ given by

$$
f^{*} \alpha=\alpha_{\mu} \circ f \frac{\partial}{\partial x^{a}}\left(\psi^{\mu} \circ f \circ \phi^{-1}\right) \circ \phi d x^{a}
$$

when expressed with respect to $(\mathcal{U}, \phi)$. The pull-back operation is extended to higher degree differential forms as a tensor homomorphism

$$
f^{*}(\alpha \wedge \beta)=f^{*} \alpha \wedge f^{*} \beta
$$

where $\beta$ is a differential form. It can be shown that the exterior derivative and pull-back operations commute

$$
f^{*} d \alpha=d f^{*} \alpha
$$




\subsection{Cubes and chains}

Let $(\mathcal{U}, \phi),[0,1]^{r} \subset \mathcal{U}$, be the natural chart on $\mathbb{R}^{r}$, i.e. $\sigma^{j}=\phi^{j}(p)$, and $0 \leq \sigma^{j} \leq 1, j \in \mathbb{Z}$. An $r$-cube $e^{4}$ on a differential manifold $\mathcal{M}$ is the pair $\left(c^{r}, \Omega^{r}\right)$ where $c^{r}:[0,1]^{r} \rightarrow \mathcal{M}$ is a smooth map and

$$
\Omega^{r}= \pm d \sigma^{j_{1}} \wedge d \sigma^{j_{2}} \wedge \ldots d \sigma^{j_{r}}, \quad j_{1}, j_{2}, \ldots j_{r} \in \mathbb{Z}
$$

is a differential $r$-form (an orientation) on $\mathbb{R}^{r}$. We will examine the choice of sign shortly.

A finite sum of $r$-cubes $\left\{\left(c_{J}^{r}, \Omega_{J}^{r}\right)\right\}$ with real coefficients $\left\{b_{J}\right\}, J \in \mathbb{Z}$, is called an r-chain.

Each $r$-cube gives rise to $2 r(r-1)$-cubes known as faces. Each face, denoted $c_{j, \varepsilon}^{r-1}$, is obtained by restricting $c^{r}$ to the points $p \in[0,1]^{r}$ such that $\sigma^{j}=\phi^{j}(p)=\varepsilon$, where $\varepsilon=0,1$. The orientation $\Omega_{j, \varepsilon}^{r-1}$ of each face is obtained from $\Omega^{r}$ by

$$
\Omega_{j, \varepsilon}^{r-1} \equiv(-1)^{\varepsilon+1} \iota_{\partial / \partial \sigma^{j}} \Omega^{r}
$$

Note that faces inherit their orientation from that of a higher-dimensional cube, which we call their parent cube. Once a parent cube is defined all of the orientations of its faces are fixed by (35). Thus, the sign in (34) must either be fixed as part of the definition of $\Omega^{r}$ if $\left(c^{r}, \Omega^{r}\right)$ is a parent cube, or inherited from its parent cube through (35).

The $r$-cube $\left(c^{r}, \Omega^{r}\right)$ has a boundary $(r-1)$-chain $\partial\left(c^{r}, \Omega^{r}\right)$,

$$
\partial\left(c^{r}, \Omega^{r}\right)=\sum_{j=1}^{r} \sum_{\varepsilon=0,1}\left(c_{j, \varepsilon}^{r-1}, \Omega_{j, \varepsilon}^{r-1}\right),
$$

where the $(r-1)$-cube in each term of the summand is a face of $\left(c^{r}, \Omega^{r}\right)$. The boundary $\partial C^{r}$ of the $r$-chain $C^{r}$

$$
C^{r}=\sum_{J} b_{J}\left(c_{J}^{r}, \Omega_{J}^{r}\right)
$$

\footnotetext{
${ }^{4}$ Technically, this is an oriented $r$-cube.
} 
is

$$
\partial C^{r} \equiv \sum_{J} b_{J} \partial\left(c_{J}^{r}, \Omega_{J}^{r}\right)
$$

\subsubsection{Example}

Let $\left(c^{2}, \Omega^{2}\right)$ be a 2-cube on a 2-dimensional manifold $\mathcal{M}$. We can always find a chart $(\mathcal{U}, \phi)$ on $\mathcal{M}$ with respect to which the components of $c^{2}$ are the identity map. The chart $(\mathcal{U}, \phi)$ is said to be adapted to $c^{2}$. Thus, with $x=\phi^{1}(p)$ and $y=\phi^{2}(p)$ at $p \in \mathcal{U}$, the map $c^{2}$ has components

$$
(x, y)=c^{2}\left(\sigma^{1}, \sigma^{2}\right)=\left(\sigma^{1}, \sigma^{2}\right) .
$$

The orientation associated with $c^{2}$ is

$$
\Omega^{2}=d \sigma^{1} \wedge d \sigma^{2}
$$

The faces of the parent cube $\left(c^{2}, \Omega^{2}\right)$ are

$$
\begin{aligned}
& (x, y)=c_{1,0}^{1}\left(0, \sigma^{2}\right)=\left(0, \sigma^{2}\right), \\
& (x, y)=c_{1,1}^{1}\left(1, \sigma^{2}\right)=\left(1, \sigma^{2}\right), \\
& (x, y)=c_{2,0}^{1}\left(\sigma^{1}, 0\right)=\left(\sigma^{1}, 0\right), \\
& (x, y)=c_{2,0}^{1}\left(\sigma^{1}, 1\right)=\left(\sigma^{1}, 1\right)
\end{aligned}
$$

with orientations

$$
\begin{aligned}
& \Omega_{1,0}^{1}=-d \sigma^{2} \\
& \Omega_{1,1}^{1}=d \sigma^{2} \\
& \Omega_{2,0}^{1}=d \sigma^{1} \\
& \Omega_{2,1}^{1}=-d \sigma^{1} .
\end{aligned}
$$


Thus, the 1-chain $\partial\left(c^{2}, \Omega^{2}\right)$ is

$$
\begin{aligned}
\partial\left(c^{2}, \Omega^{2}\right)= & \left(c_{1,0}^{1},-d \sigma^{2}\right)+\left(c_{1,1}^{1}, d \sigma^{2}\right) \\
& +\left(c_{2,0}^{1}, d \sigma^{1}\right)+\left(c_{2,1}^{1},-d \sigma^{1}\right) .
\end{aligned}
$$

\subsection{Integration and Stokes' theorem}

Any differential $r$-form $\omega$ on $\mathbb{R}^{r}$ can be written

$$
\omega=f \Omega^{r}
$$

where $f$ is a smooth function on $\mathbb{R}^{r}$ and

$$
\Omega^{r}= \pm d \sigma^{j_{1}} \wedge d \sigma^{j_{2}} \wedge \ldots d \sigma^{j_{r}}
$$

The integral of $\omega$ over $[0,1]^{r}$ is defined to be

$$
\begin{aligned}
\int_{[0,1]^{r}} \omega & =\int_{[0,1]^{r}} f\left(\sigma^{j_{1}}, \sigma^{j_{2}}, \ldots \sigma^{j_{r}}\right) \Omega^{r} \\
& \equiv \int_{0}^{1} \int_{0}^{1} \ldots \int_{0}^{1} f\left(\sigma^{j_{1}}, \sigma^{j_{2}}, \ldots \sigma^{j_{r}}\right) d \sigma^{j_{1}} d \sigma^{j_{2}} \ldots d \sigma^{j_{r}}
\end{aligned}
$$

regardless of the choice of sign in $\Omega^{r}$. The integral of a differential $r$-form $\alpha$ on any manifold $\mathcal{M}$ over the $r$-cube $\left(c^{r}, \Omega^{r}\right)$ is defined via the pull-back map $c^{r *}$. The $r$-form $c^{r *} \alpha$ on $\mathbb{R}^{r}$ is, with respect to the chart $(\mathcal{U}, \phi), \sigma=\phi^{j}(p)$ at $p \in \mathbb{R}^{r}$,

$$
c^{r *} \alpha=h \Omega^{r}
$$

where $h$ is a smooth function on $\mathbb{R}^{r}$. We define

$$
\int_{c^{r}} \alpha \equiv \int_{[0,1]^{r}} c^{r *} \alpha
$$


and so using (37)

$$
\begin{aligned}
\int_{[0,1]^{r}} c^{r *} \alpha & =\int_{[0,1]^{r}} h\left(\sigma^{j_{1}}, \sigma^{j_{2}}, \ldots \sigma^{j_{r}}\right) \Omega^{r} \\
& =\int_{[0,1]^{r}} h\left(\sigma^{j_{1}}, \sigma^{j_{2}}, \ldots \sigma^{j_{r}}\right) d \sigma^{j_{1}} d \sigma^{j_{2}} \ldots d \sigma^{j_{r}}
\end{aligned}
$$

The integral of $\alpha$ over

$$
C^{r}=\sum_{J} b_{J}\left(c_{J}^{r}, \Omega_{J}^{r}\right)
$$

is

$$
\int_{C^{r}} \alpha \equiv \sum_{J} b_{J} \int_{c_{J}^{r}} \alpha
$$

This formalism leads to the remarkably beautiful result known as the Newton-Leibniz-Gauss-Green-Ostrogradskii-Stokes-Poincaré theorem, or Stokes' theorem for short,

$$
\int_{C^{r}} d \alpha=\int_{\partial C^{r}} \alpha
$$

Thus, using (22) we have an analogue of the "integration by parts" formula,

$$
\int_{C^{r}} d \alpha \wedge \beta=\int_{\partial C^{r}} \alpha \wedge \beta-(-1)^{p} \int_{C^{r}} \alpha \wedge d \beta
$$

where $\alpha$ is a differential $p$-form. For notational simplicity, although it is an abuse of the notation, if an $r$-chain consists of only one $r$-cube then we shall use the same label for the $r$-cube map as used for the $r$-chain. 


\subsection{Example}

Let $(\mathcal{U}, \phi)$ be a spherical polar chart on $\mathcal{M}=\mathbb{R}^{3}$, i.e. one in which the metric has the form

$$
g=d r \otimes d r+r^{2}\left(d \theta \otimes d \theta+\sin ^{2} \theta d \varphi \otimes d \varphi\right) .
$$

Thus,

$$
\begin{aligned}
& e^{1}=d r \\
& e^{2}=r d \theta \\
& e^{3}=r \sin \theta d \varphi
\end{aligned}
$$

is an orthonormal co-frame. Let us choose the orientation

$$
\begin{aligned}
\star 1 & =e^{1} \wedge e^{2} \wedge e^{3} \\
& =r^{2} \sin \theta d r \wedge d \theta \wedge d \varphi .
\end{aligned}
$$

Let $\Sigma$ be a 2-chain on $\mathbb{R}^{3}$ consisting of only one 2 -cube with

$$
\begin{aligned}
& r=1 \\
& \theta=\Sigma^{1}(p)=\pi \sigma^{1}, \\
& \varphi=\Sigma^{2}(p)=2 \pi \sigma^{2}
\end{aligned}
$$

and orientation $\Omega^{2}=d \sigma^{1} \wedge d \sigma^{2}$. The pull-back with respect to $\Sigma$ of each element of the cobasis $\{d r, d \theta, d \phi\}$ is

$$
\begin{aligned}
& \Sigma^{*} d r=d \Sigma^{*} r=d 1=0, \\
& \Sigma^{*} d \theta=d \Sigma^{*} \theta=\pi d \sigma^{1}, \\
& \Sigma^{*} d \phi=d \Sigma^{*} \phi=2 \pi d \sigma^{2}
\end{aligned}
$$

using (33). The vector field $\partial / \partial r$ is normal to the image set $\mathcal{D}_{\Sigma}$ of $\Sigma$, i.e.

$$
\begin{aligned}
\Sigma^{*}(g(\partial / \partial r,-)) & =\Sigma^{*} d r \\
& =0
\end{aligned}
$$


A volume form on $\mathcal{D}_{\Sigma}$ induced from $\star 1$ is

$$
\# 1=\iota_{\partial} / \partial r \star 1
$$

and so the area of $\mathcal{D}_{\Sigma}$ is

$$
\begin{aligned}
\int_{\Sigma} \# 1 & =\int_{[0,1]^{2}} \Sigma^{*} \# 1 \\
& =\int_{[0,1]^{2}} \Sigma^{*}(\sin \theta d \theta \wedge d \varphi) \\
& =2 \pi^{2} \int_{[0,1]^{2}} \sin \left(\pi \sigma^{1}\right) d \sigma^{1} \wedge d \sigma^{2} \\
& =2 \pi^{2} \int_{[0,1]^{2}}^{1} \sin \left(\pi \sigma^{1}\right) \Omega^{2} \\
& =2 \pi^{2} \int_{0}^{1} \int_{0}^{1} \sin \left(\pi \sigma^{1}\right) d \sigma^{1} d \sigma^{2} \\
& =4 \pi .
\end{aligned}
$$

Note that the "outward" pointing normal (i.e. that which points away from the coordinate singularity at $r=0$ ) was used to construct \#1. An alternative (although less conventional) choice is to use the "inward" pointing normal $-\partial / \partial r$.

\section{Standard vector calculus in terms of exterior calculus}

Let us focus on the special case $\mathcal{M}=\mathbb{R}^{3}$ endowed with the standard Euclidean metric. This means there exists a global chart $\left(\mathbb{R}^{3}, \phi\right)$, where 
$\left\{x^{a}=\phi^{a}(p)\right\}, p \in \mathbb{R}^{3}$, with respect to which the metric tensor $g$ has the form

$$
g=d x^{1} \otimes d x^{1}+d x^{2} \otimes d x^{2}+d x^{3} \otimes d x^{3} .
$$

Such charts on $\mathbb{R}^{3}$ are called Cartesian. We choose the orientation

$$
\star 1=d x^{1} \wedge d x^{2} \wedge d x^{3}
$$

and note that, using (29),

$$
\star^{2} \alpha=\alpha
$$

for any degree differential form $\alpha$ on $\mathbb{R}^{3}$. Thus

$$
{ }^{-1} \alpha=\star \alpha \text {. }
$$

To see this observe that $\{\partial / \partial x, \partial / \partial y, \partial / \partial z\}$ is an orthonormal frame and that, in the notation of $(29)$, $\operatorname{det} \boldsymbol{\eta}=1$. Furthermore, the values of $p(3-p)$ for each $p \in\{0,1,2,3\}$ are all even.

Let $\{\boldsymbol{i}, \boldsymbol{j}, \boldsymbol{k}\}$ be the unit orthonormal vector basis, in the conventional sense, corresponding to $\{\partial / \partial x, \partial / \partial y, \partial / \partial z\}$. This means that given a conventional vector field $\boldsymbol{u}$ we construct a vector field $U$ on $\mathbb{R}^{3}$ considered as a manifold by the following replacements :

$$
\begin{aligned}
& \boldsymbol{i} \rightarrow \partial / \partial x \\
& \boldsymbol{j} \rightarrow \partial / \partial y \\
& \boldsymbol{k} \rightarrow \partial / \partial z
\end{aligned}
$$

Thus, if

$$
\boldsymbol{u}=a(x, y, z) \boldsymbol{i}+b(x, y, z) \boldsymbol{j}+c(x, y, z) \boldsymbol{k}
$$

then $U$, with respect to the chart $\left(\mathbb{R}^{3}, \phi\right)$, has the form

$$
U=a(x, y, z) \frac{\partial}{\partial x}+b(x, y, z) \frac{\partial}{\partial y}+c(x, y, z) \frac{\partial}{\partial z} .
$$

We will indicate this correspondence as equality, i.e.

$$
U=\boldsymbol{u} .
$$




\subsection{Dot and cross products}

Let $\boldsymbol{u}$ and $\boldsymbol{v}$ be conventional vector fields on $\mathbb{R}^{3}$ and their corresponding vector fields on $\mathbb{R}^{3}$, considered as a manifold, be $U=\boldsymbol{u}$ and $V=\boldsymbol{v}$. Then, the conventional dot product $\boldsymbol{u} \cdot \boldsymbol{v}$ is

$$
\boldsymbol{u} \cdot \boldsymbol{v}=g(U, V)
$$

whilst the conventional cross product $\boldsymbol{u} \times \boldsymbol{v}$ is

$$
\boldsymbol{u} \times \boldsymbol{v}=g^{-1}(\star(\tilde{U} \wedge \tilde{V}),-)
$$

The cyclic symmetry of the triple vector product

$$
\boldsymbol{u} \cdot(\boldsymbol{v} \times \boldsymbol{w})=\boldsymbol{v} \cdot(\boldsymbol{w} \times \boldsymbol{u})=\boldsymbol{w} \cdot(\boldsymbol{u} \times \boldsymbol{v})
$$

follows as a consequence of the properties of the exterior product

$$
\begin{aligned}
\boldsymbol{u} \cdot(\boldsymbol{v} \times \boldsymbol{w}) & =g^{-1}(\star(\tilde{U} \wedge \tilde{V} \wedge \tilde{W}),-) \\
& =g^{-1}(\star(\tilde{V} \wedge \tilde{W} \wedge \tilde{U}),-) \\
& =g^{-1}(\star(\tilde{W} \wedge \tilde{U} \wedge \tilde{V}),-)
\end{aligned}
$$

where $W=\boldsymbol{w}$.

\subsection{Grad, curl and div}

The operations grad,curl and div are

$$
\begin{aligned}
& \operatorname{grad}(f)=\frac{\tilde{d f},}{\operatorname{curl}(\boldsymbol{u})=\star d \tilde{U},} \\
& \operatorname{div}(\boldsymbol{u})=\star \star-1 d \tilde{U}
\end{aligned}
$$


in exterior form, where $f$ is a smooth function on $\mathbb{R}^{3}$. All of the wellknown identities involving these three operations can be obtained in a straightforward manner using the material in section 3. For example

$$
\begin{aligned}
\widetilde{\star d^{2} f} & =0 \\
& =\operatorname{curl}(\operatorname{grad}(f)),
\end{aligned}
$$

using (23) and

$$
\begin{aligned}
\operatorname{div}(\boldsymbol{u} \times \boldsymbol{v}) & ={ }^{-1} d \star(\star(\tilde{U} \wedge \tilde{V})) \\
& ={ }^{-1} d(\tilde{U} \wedge \tilde{V}) \\
& ={ }^{-1}(d \tilde{U} \wedge \tilde{V}-\tilde{U} \wedge d \tilde{V}) \\
& =\iota_{V} \star d \tilde{U}-\iota_{U} \star d \tilde{V} \\
& =g(\widetilde{\star d \tilde{U}}, V)-g(\widetilde{\star d \tilde{V}}, U) \\
& =\boldsymbol{v} \cdot \operatorname{curl}(\boldsymbol{u})-\boldsymbol{u} \cdot \operatorname{curl}(\boldsymbol{v})
\end{aligned}
$$

using (43), (39), (22), (40), (27), (24) and (42).

\subsection{Integral relations}

Let $\Omega:[0,1]^{3} \rightarrow \mathbb{R}^{3}$ and $\Sigma:[0,1]^{2} \rightarrow \mathbb{R}^{3}$ be an oriented 3-chain and 2-chain respectively. Let their image sets be labelled $\mathcal{D}_{\Omega}$ and $\mathcal{D}_{\Sigma}$. Let the outward pointing normal of the image set $\mathcal{D}_{C^{2}}$ of a 2-chain $C^{2}$ be labelled $\boldsymbol{n}_{C^{2}}$ as a conventional vector field and $N_{C^{2}}$ as a vector field on $\mathbb{R}^{3}$ as a manifold. Let $d \mu^{3}, d \mu_{C^{2}}^{2}$ be integration measures such that

$$
\begin{aligned}
& \int_{\mathcal{D}_{\Omega}} f d \mu^{3}=\int_{\Omega} f \star 1, \\
& \int_{\mathcal{D}_{C^{2}}} f d \mu_{C^{2}}^{2}=\int_{C^{2}} f \star \tilde{N}_{C^{2}}
\end{aligned}
$$


where $f$ is any smooth function. The traditional Stokes' theorem

$$
\int_{\mathcal{D}_{\Sigma}} \operatorname{curl}(\boldsymbol{v}) \cdot \boldsymbol{n}_{\Sigma} d \mu_{\Sigma}^{2}=\int_{\mathcal{D}_{\partial \Sigma}} \boldsymbol{v} \cdot d \boldsymbol{r}
$$

and divergence theorem

$$
\int_{\mathcal{D}_{\Omega}} \operatorname{div}(\boldsymbol{v}) d \mu^{3}=\int_{\mathcal{D}_{\partial \Omega}} \boldsymbol{v} \cdot \boldsymbol{n}_{\partial \Omega} d \mu_{\partial \Omega}^{2},
$$

are consequences of (38) with $C^{r}=\Sigma$ and $C^{r}=\Omega$ respectively.

Let us first focus on (47). We note that

$$
\begin{aligned}
\int_{\mathcal{D}_{\Omega}} \operatorname{div}(\boldsymbol{u}) d \mu^{3} & =\int_{\Omega}(\star d \star \tilde{U}) \star 1 \\
& =\int_{\Omega} \star(\star x d \star \tilde{U}) \\
& =\int_{\Omega} d \star \tilde{U} \\
& =\int_{\partial \Omega} \star \tilde{U} .
\end{aligned}
$$

To go further we need to examine the integrand in (48). Any differential 1-form $\alpha$ on $\mathbb{R}^{3}$ can be written in the form

$$
\begin{aligned}
& \alpha=\alpha\left(N_{C^{2}}\right) \tilde{N}_{C^{2}}+\beta \\
& \iota_{N_{C^{2}}} \beta=0
\end{aligned}
$$

and since

$$
\iota_{N_{C^{2}}} \beta \star 1=\tilde{N}_{C_{2}} \wedge \star \beta
$$

using (30) we note that

$$
\tilde{N}_{C_{2}} \wedge \star \beta=0
$$


implying that

$$
\star \beta=\tilde{N}_{C_{2}} \wedge \gamma
$$

where $\gamma$ is a differential 1-form. Since

$$
C^{2 *} \tilde{N}_{C^{2}}=C^{2 *}\left[\tilde{N}_{C^{2}}\left(X_{\alpha}\right) e^{\alpha}\right]=0
$$

where $\left\{X_{\alpha}, N_{C^{2}}\right\}, \alpha=1,2$, is a frame adapted to $C^{2}$ with dual coframe $\left\{e^{\alpha}, \tilde{N}_{C^{2}}\right\}$, it follows that using (32)

$$
C^{2 *} \star \beta=0
$$

Therefore,

$$
C^{2 *} \star \alpha=C^{2 *}\left[\alpha\left(N_{C^{2}}\right) \star \tilde{N}_{C^{2}}\right] .
$$

Continuing with (48) we find that

$$
\begin{aligned}
\int_{\mathcal{D}_{\Omega}} \operatorname{div}(\boldsymbol{u}) d \mu^{3} & =\int_{\partial \Omega} \star \tilde{U} \\
& =\int_{\partial \Omega} g\left(U, N_{\partial \Omega}\right) \star \tilde{N}_{C^{2}} \\
& =\int_{\mathcal{D}_{\partial \Omega}} \boldsymbol{u} \cdot \boldsymbol{n} d \mu_{\partial \Omega}^{2}
\end{aligned}
$$

which is the conventional divergence theorem. Equation (49) is used in the penultimate step. 
Similarly, focussing on (46) we obtain the conventional Stokes' theorem

$$
\begin{aligned}
\int_{\mathcal{D}_{\Sigma}} \operatorname{curl}(\boldsymbol{v}) \cdot \boldsymbol{n}_{\Sigma} d \mu_{\Sigma}^{2} & =\int_{\Sigma}\left(\iota_{N_{\Sigma}}{ }^{-1} d \tilde{V}\right) \star \tilde{N}_{\Sigma} \\
& =\int_{\Sigma} \star\left(\tilde{N}_{\Sigma} \iota_{N_{\Sigma}}{ }^{-1} d \tilde{V}\right) \\
& =\int_{\Sigma} \star(\star d \tilde{V}) \\
& =\int_{\Sigma} d \tilde{V} \\
& =\int_{\partial \Sigma} \tilde{V} \\
& =\int_{[0,1]}(\partial \Sigma)^{*} \tilde{V} \\
& =\int_{0}^{1} V_{a} \circ \partial \Sigma(\sigma) \frac{d \partial \Sigma^{a}}{d \sigma}(\sigma) d \sigma \\
& =\int_{0}^{1} \boldsymbol{v}(\sigma) \cdot \frac{d \boldsymbol{r}}{d \sigma}(\sigma) d \sigma \\
& =\int_{\mathcal{D}_{\partial \Sigma}} \boldsymbol{v} \cdot d \boldsymbol{r}
\end{aligned}
$$

where $V_{a}=\tilde{V}\left(\partial / \partial x^{a}\right)$ and $\boldsymbol{r}(\sigma)$ is the position vector that locates the point labelled by $\sigma$ on $\mathcal{D}_{\partial \Sigma}$, i.e.

$$
\boldsymbol{r}(\sigma)=\partial \Sigma^{a}(\sigma) \frac{\partial}{\partial x^{a}}
$$

\subsection{Applications involving Stokes' theorem on $\mathbb{R}^{3}$}

Note the plethora of metric and Hodge operations that occur when comparing conventional vectorial equations with their equivalent dif- 
ferential form equations. The reason is that we have insisted on replacing conventional vector field, for example $\boldsymbol{u}$, with their differential geometric vector field counterparts, for example $U$. However, this is often not the best strategy to adopt. The formalism is at its most powerful when it is recognized that many vectorial quantities are more efficiently represented by differential forms. For example, if $V$ is the velocity of a fluid flow on $\mathbb{R}^{3}$ then it is natural to work with the vorticity 2-form $\omega$

$$
\omega=d \tilde{V}
$$

rather than the vector field $W$

$$
W=\widetilde{\star d \tilde{V}} .
$$

The vorticity $\Gamma[\Sigma]$ across a 2-chain $\Sigma$ is simply

$$
\begin{aligned}
\Gamma[\Sigma] & \equiv \int_{\Sigma} \omega \\
& =\int_{\Sigma} d \tilde{V} \\
& =\int_{\partial \Sigma} \tilde{V}
\end{aligned}
$$

which shows that the vorticity is just the circulation around the 1chain $\partial \Sigma$.

Another nice example is to consider the electric field of an isolated static point charge. Traditionally, one thinks in terms of an electric field vector $\boldsymbol{E}$ and an electric potential $\Phi$ where $\boldsymbol{E}=-\operatorname{grad}(\Phi)$. Here, it is more natural to think in terms of an electric differential 1-form $E=-d \Phi$ (where $\boldsymbol{E}=\tilde{E}$ ) on the 3-dimensional differential manifold $(\mathcal{M}, g), \mathcal{M}=\mathbb{R}^{3}-\{p\}$ where $p$ is the location of the point source. That $E$ is divergence-free means that

$$
d \star E=0
$$




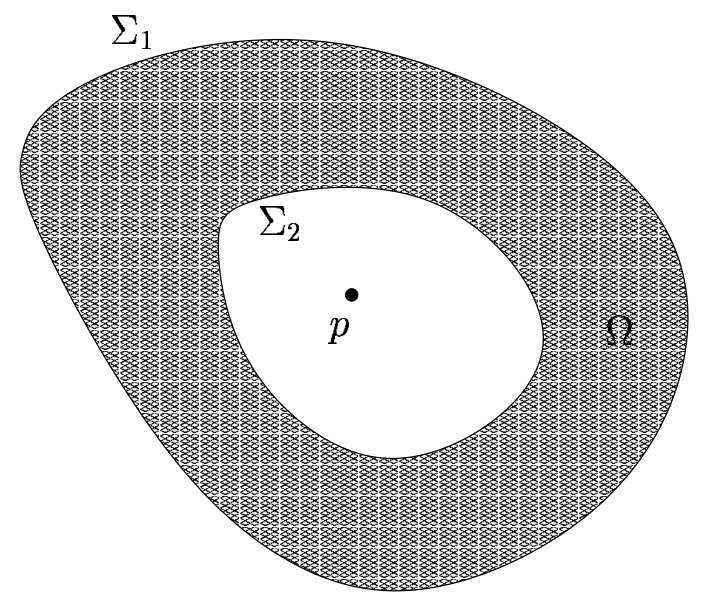

Figure 3: A schematic diagram of the chains used in this section. The image set of the 3-chain $\Omega$ is the shaded region. The boundary 2-chain of $\Omega$ is $\partial \Omega=\Sigma_{1}+\Sigma_{2}$. The point $p$ is not in $\mathcal{M}$ so there exist closed 2-chains that are not contractible to $p$.

and Laplace's equation is obtained when we substitute $E$ with $-d \Phi$,

$$
d \star d \Phi=0 .
$$

Let $\Omega$ be a 3-chain on $\mathcal{M}$ whose boundary 2-chain $\partial \Omega$ is $\partial \Omega=\Sigma_{1}+\Sigma_{2}$ where $\left\{\Sigma_{1}, \Sigma_{2}\right\}$ are closed 2-cubes (see figure 3 ). Then

$$
\begin{aligned}
\int_{\Omega} d \star E & =\int_{\partial \Omega} \star E \\
& =\int_{\Sigma_{1}} \star E+\int_{\Sigma_{2}} \star E
\end{aligned}
$$

and so

$$
\int_{\Sigma_{1}} \star E=-\int_{\Sigma_{2}} \star E .
$$


Since the normals to the images of the 2-chains $\Sigma_{1}$ and $\Sigma_{2}$ are oppositely oriented we conclude that

$$
P[\Sigma] \equiv \int_{\Sigma} \star E
$$

is the same for all $\Sigma$ topologically equivalent to $\Sigma_{1}$. The Poincaré lemma tells us that that $\star E=d \alpha$ on an open subset $\mathcal{U} \subset \mathcal{M}$ where $\alpha$ is a differential 1-form on $\mathcal{U}$. However $\star E$ is not exact, i.e. cannot be written $d \alpha$ where $\alpha$ is a differential form on all of $\mathcal{M}$ and so $P[\Sigma]$ is in general non-zero.

The number $P[\Sigma]$ is known as the de Rham period of $\star E$ over $\Sigma$ and, physically, it is the electric charge of the point $p$. In a spherical polar chart $(\mathcal{U}, \psi)$ with $p$ located at $r=0$ the metric tensor has the form

$$
g=d r \otimes d r+r^{2} d \theta \otimes d \theta+r^{2} \sin ^{2} \theta . d \varphi \otimes d \varphi,
$$

A solution to Laplace's equation on $\mathcal{M}$ is

$$
\Phi(r, \theta, \varphi)=\frac{c}{r}
$$

where $c$ is a constant scalar. To see this, choose the orientation

$$
\star 1=+e^{1} \wedge e^{2} \wedge e^{3}
$$

where $\left\{e^{1}, e^{2}, e^{3}\right\}$ is the orthonormal co-frame

$$
\begin{aligned}
e^{1} & =d r, \\
e^{2} & =r d \theta, \\
e^{3} & =r \sin \theta d \varphi .
\end{aligned}
$$


Thus,

$$
\begin{aligned}
\star d \Phi & =\star d \frac{c}{r}, \\
& =-\star \frac{c}{r^{2}} d r \\
& =-\frac{c}{r^{2}} \star e^{1} \\
& =-\frac{c}{r^{2}} e^{2} \wedge e^{3}, \\
& =-c \sin \theta d \theta \wedge d \varphi
\end{aligned}
$$

and so, as promised, $d \star d \Phi=0$ on $\mathcal{M}$. The de Rham period of $\star d \Phi$ over the 2-chain $\left(\Sigma, d \sigma^{1} \wedge d \sigma^{2}\right)$ with components $\left\{r=r_{0}, \theta=\pi \sigma^{1}, \varphi=\right.$ $\left.2 \pi \sigma^{2}\right\}$ is

$$
\begin{aligned}
\int_{\Sigma} \star d \Phi & =\int_{\Sigma}-c \sin \theta d \theta \wedge d \varphi \\
& =-4 \pi c .
\end{aligned}
$$

Introducing the charge $q \equiv-4 \pi c$ of the source we see that

$$
\Phi(r, \theta, \varphi)=-\frac{q}{4 \pi r}
$$

which is the electric potential of an electric monopole of charge $q$ at $p$.

\section{Differential operators on tensor bun- dles}

So far the only differential operator that we have discussed is the exterior derivative $d$ on the bundle of differential forms. It is also very useful to be able to differentiate arbitrary type tensors, which is the 
focus of this section. We will discuss two types of differential operators on tensors bundles : the Lie derivative and linear connections. So far we have only considered vector fields in terms of derivations on $\mathcal{F}(\mathcal{M})$. Before we can discuss the Lie derivative we need to introduce the notion of the flow of a vector field.

\subsection{The push-forward map}

Recall that a smooth map $f: \mathcal{M} \rightarrow \mathcal{N}$ between two manifolds $\mathcal{M}$ and $\mathcal{N}$ induces the pull-back map $f^{*}: \Lambda \mathcal{N} \rightarrow \Lambda \mathcal{M}$ (see section 4.1). If $f$ is one-to-one then it also induces the push-forward map $f_{*}: T \mathcal{M} \rightarrow T \mathcal{N}$ between the tangent bundles of $\mathcal{M}$ and $\mathcal{N}$. Let $X \in \Gamma T \mathcal{M}$ be a vector field on $\mathcal{M}$ and define the push-forward of $X$ with respect to $f$, denoted by $f_{*} X$, via

$$
f^{*}\left[\alpha\left(f_{*} X\right)\right]=\left(f^{*} \alpha\right)(X)
$$

where $\alpha \in \Gamma \Lambda_{1} \mathcal{N}$ is any differential 1 -form on $\mathcal{N}$. In particular if we choose $\alpha=d h$ we find

$$
\begin{aligned}
f^{*}\left[d h\left(f_{*} X\right)\right] & =\left(f^{*} d h\right)(X) \\
& =d\left(f^{*} h\right)(X) \\
& =X\left(f^{*} h\right)
\end{aligned}
$$

but, on the other hand,

$$
f^{*}\left[d h\left(f_{*} X\right)\right]=f^{*}\left[\left(f_{*} X\right)(h)\right]
$$

and so we obtain the action of $f_{*} X$ on any $h \in \mathcal{F}(\mathcal{N})$ :

$$
f^{*}\left[\left(f_{*} X\right)(h)\right]=X\left(f^{*} h\right) .
$$




\subsection{One-parameter families of diffeomorphisms}

Let $\mathcal{M}$ be a differential manifold, $\mathcal{V} \subset \mathcal{M}$ be an open subset of $\mathcal{M}$ and $I \subset \mathbb{R}$ be an open interval about $0 \in \mathbb{R}$. Let $\varphi$ be a map such that

$$
\begin{aligned}
\varphi & : I \times \mathcal{V} \rightarrow \mathcal{M} \\
& (t, p) \rightarrow q=\varphi(t, p) \equiv \varphi_{t}(p)
\end{aligned}
$$

where, for each $t \in I, \varphi_{t}$ is a local diffeomorphism (a smooth map with smooth inverse) of $\mathcal{V}$ to another open subset of $\mathcal{M}$. We demand that for any pair $t_{1}, t_{2} \in I$ such that $\left(t_{1}+t_{2}\right) \in I$

$$
\varphi_{t_{2}} \circ \varphi_{t_{1}}=\varphi_{t_{1}+t_{2}}
$$

and

$$
\varphi_{0}(p)=p \quad \forall p \in \mathcal{M}
$$

Note that in particular $\varphi_{t}^{-1}=\varphi_{-t}$. The collection of maps $\left\{\varphi_{t}\right\}$ is known as a one-parameter family of local diffeomorphisms and induces a curve (a 1-chain) $C_{p}$ for each $p \in \mathcal{M}$

$$
\begin{aligned}
C_{p}: I & \rightarrow \mathcal{M} \\
& t \rightarrow \varphi_{t}(p) .
\end{aligned}
$$

The push-forward $C_{p *} \partial_{t}$ (where $\left.\partial_{t} \equiv \partial / \partial t\right)$ when evaluated at $t=0$ yields a tangent vector $X_{p} \in T_{p} \mathcal{M}$ at $p$

$$
X_{p}=\left.C_{p *} \partial_{t}\right|_{t=0} .
$$

Since $\varphi_{t}$ is smooth the set $\left\{X_{p}\right\}$ leads to the vector field $X \in \Gamma T \mathcal{M}$ given by

$$
\left.X\right|_{p} \equiv X_{p}
$$


and so a one-parameter family of local diffeomorphisms on $\mathcal{M}$ induces a vector field on $\mathcal{M}$. Conversely, given a vector field $X \in \Gamma T \mathcal{M}$ one can generate a one-parameter family $\left\{\psi_{t}\right\}$ of local diffeomorphisms of $\mathcal{M}$ by solving for a set of integral curves $\left\{\mathcal{C}_{p}\right\}$ of $X$ (also known as the flow of $X$ )

$$
\begin{aligned}
\mathcal{C}_{p}: I & \rightarrow \mathcal{M} \\
t & \rightarrow q=\mathcal{C}_{p}(t) \\
\mathcal{C}_{p *} \partial_{t} & =X \\
\mathcal{C}_{p}(0) & =p
\end{aligned}
$$

and then defining

$$
\psi_{t}(p) \equiv \mathcal{C}_{p}(t) .
$$

With respect to a local chart $(\mathcal{U}, \phi)$, with coordinates $\left\{x^{a}\right\}$, the above becomes

$$
\begin{aligned}
\mathcal{C}_{p}^{*}\left[d x^{a}\left(\mathcal{C}_{p *} \partial_{t}\right)\right] & =d \mathcal{C}_{p}^{a} / d t \\
& =\mathcal{C}_{p}^{*}\left[d x^{a}(X)\right] \\
& =\xi^{a} \circ \mathcal{C}_{p}
\end{aligned}
$$

where $\left\{\xi^{a}\right\}$ and $\left\{\mathcal{C}_{p}^{a}\right\}$ are the components of $X$ and $\mathcal{C}_{p}$ with respect to $(\mathcal{U}, \phi)$. Equations $\mathcal{C}_{p *} \partial_{t}=X$ and $C_{p}(0)=p$ translated into a differential equation for $\psi$ read

$$
\frac{d \psi^{a}}{d t}(t, p)=\left(\xi^{a} \circ \psi\right)(t, p)
$$

subject to the initial condition

$$
\psi^{a}(0, p)=x_{0}^{a}
$$

where $x_{0}^{a}=\phi^{a}(p)$ is the coordinate representation of $p \in \mathcal{M}$ with respect $(\mathcal{U}, \phi)$. 


\subsubsection{Example}

Let $X \in \Gamma T \mathcal{M}, \operatorname{dim} \mathcal{M}=2$, be the vector field

$$
X=y \frac{\partial}{\partial x}-x \frac{\partial}{\partial y}
$$

with respect to a chart $(\mathcal{U}, \phi)$ with coordinates $\{x, y\}$. An integral curve $C:[0,1] \rightarrow \mathcal{M}$ of $X$ is a solution to

$$
C_{*} \partial_{t}=X
$$

or, in componential form where $\left\{x=C^{1}(t), y=C^{2}(t)\right\}$,

$$
\begin{aligned}
& \frac{d C^{1}}{d t}=C^{2}(t), \\
& \frac{d C^{2}}{d t}=-C^{1}(t)
\end{aligned}
$$

which with the initial condition

$$
\begin{aligned}
& C^{1}(0)=a, \\
& C^{2}(0)=b,
\end{aligned}
$$

has the solution

$$
\begin{aligned}
& C^{1}(t)=a \cos (t)+b \sin (t), \\
& C^{2}(t)=-a \sin (t)+b \cos (t) .
\end{aligned}
$$

Thus $X$ induces a one-parameter family of local diffeomorphisms $\left\{\psi_{t}\right\}$ whose coordinate expressions are

$$
\begin{aligned}
& \psi_{t}^{1}(x, y)=x \cos (t)+y \sin (t) \\
& \psi_{t}^{2}(x, y)=-x \sin (t)+y \cos (t)
\end{aligned}
$$




\subsection{The Lie derivative}

The notion of one-parameter families of local diffeomorphisms of $\mathcal{M}$ leads very naturally to a type-preserving derivation on tensor fields known as the Lie derivative. Let $\left\{\varphi_{t}\right\}$ be a one-parameter family of local diffeomorphisms of $\mathcal{M}$ with $p=\varphi_{0}(p)$ and define the oneparameter family of maps $\left\{\hat{\varphi}_{t}\right\}$ by

$$
\begin{aligned}
& \hat{\varphi}_{t} \alpha=\varphi_{t}^{*} \alpha, \\
& \hat{\varphi}_{t} X=\varphi_{-t *} X, \\
& \hat{\varphi}_{t}(S \otimes T)=\hat{\varphi}_{t} S \otimes \hat{\varphi}_{t} T
\end{aligned}
$$

where $\alpha$ is a differential form, $X$ is a vector field and $S$ and $T$ are arbitrary type tensors on $\mathcal{M}$. The Lie derivative $\mathcal{L}_{X} T$ of a tensor $T$ at $p \in \mathcal{M}$ with respect to the vector field $X$ induced from $\left\{\varphi_{t}\right\}$ is

$$
\mathcal{L}_{X} T(p) \equiv \lim _{t \rightarrow 0} \frac{1}{t}\left(\hat{\varphi}_{t} T-\hat{\varphi}_{0} T\right)(p) .
$$

For example, for $f \in \Gamma \Lambda_{0} \mathcal{M}$ (i.e. $f \in \mathcal{F}(\mathcal{M})$ )

$$
\begin{aligned}
\mathcal{L}_{X} f(p) & =\lim _{t \rightarrow 0} \frac{1}{t}\left(\varphi_{t}^{*} f-\varphi_{0}^{*} f\right)(p) \\
& =\lim _{t \rightarrow 0} \frac{1}{t}\left\{f\left[\varphi_{t}(p)\right]-f(p)\right\} \\
& =\left.\left(C_{p *} \partial_{t}\right)(f)\right|_{t=0} \\
& =X f(p)
\end{aligned}
$$

i.e.

$$
\mathcal{L}_{X} f=X f .
$$

It can also be shown that

$$
\left(\mathcal{L}_{X} Y\right) f(p)=X(Y f)(p)-Y(X f)(p)
$$


or

$$
\mathcal{L}_{X} Y=[X, Y]
$$

where $Y$ is a vector field and the commutator $[X, Y] \equiv X Y-Y X$ is known as the Lie bracket of $X$ and $Y$. Let $\left\{x^{a}\right\}$ be the coordinates of a chart $(\mathcal{U}, \phi)$ on $\mathcal{M}$ with associated local coordinate basis $\left\{\partial_{a}=\partial / \partial x^{a}\right\}$ for $\Gamma T \mathcal{M}$. Then

$$
\begin{aligned}
{[X, Y] f } & =X(Y f)-Y(X f) \\
& =\xi^{a} \partial_{a}\left(\zeta^{b} \partial_{b} f\right)-\zeta^{a} \partial_{a}\left(\xi^{b} \partial_{b} f\right) \\
& =\left(\xi^{a} \partial_{a} \zeta^{b}-\zeta^{a} \partial_{a} \xi^{b}\right) \partial_{b} f
\end{aligned}
$$

where $X=\xi^{a} \partial_{a}$ and $Y=\zeta^{a} \partial_{a}$ and the last line is obtained because $\partial_{a} \partial_{b} f=\partial_{b} \partial_{a} f$. Therefore, a coordinate expression for the Lie bracket on $\mathcal{U}$ is

$$
[X, Y]=\left(\xi^{a} \partial_{a} \zeta^{b}-\zeta^{a} \partial_{a} \xi^{b}\right) \partial_{b}
$$

It can be shown that, when applied to differential forms, the Lie derivative has the representation

$$
\mathcal{L}_{X} \alpha=d \iota_{X} \alpha+\iota_{X} d \alpha
$$

where $\alpha$ is a differential form on $\mathcal{M}$. Equation (57) is known as Cartan's identity. More generally, $\mathcal{L}_{X}$ is a type-preserving

$$
T \in \Gamma \mathbf{T}_{q}^{p} \mathcal{M} \Rightarrow \mathcal{L}_{X} T \in \Gamma \mathbf{T}_{q}^{p} \mathcal{M}
$$

derivation on tensor fields

$$
\mathcal{L}_{X}(S \otimes T)=\mathcal{L}_{X} S \otimes T+S \otimes \mathcal{L}_{X} T
$$

where $S$ and $T$ are arbitrary type tensors on $\mathcal{M}$, that commutes with contractions

$$
\mathcal{L}_{X}[\alpha(Y)]=\left(\mathcal{L}_{X} \alpha\right)(Y)-\alpha\left(\mathcal{L}_{X} Y\right)
$$


where $\alpha$ is a differential 1 -form and $Y$ is a vector field on $\mathcal{M}$, commutes with the exterior derivative $d$

$$
\mathcal{L}_{X} d=d \mathcal{L}_{X}
$$

on differential forms on $\mathcal{M}$ and satisfies

$$
\begin{aligned}
{\left[\mathcal{L}_{X}, \mathcal{L}_{Y}\right] } & \equiv \mathcal{L}_{X} \mathcal{L}_{Y}-\mathcal{L}_{Y} \mathcal{L}_{X} \\
& =\mathcal{L}_{[X, Y]} .
\end{aligned}
$$

It turns out that (54), (56), (58) and (59) are enough to specify $\mathcal{L}_{X}$ uniquely : the Lie derivative is the unique type-preserving derivation on tensor fields that commutes with contractions and satisfies (56). Finally, the Lie derivative also commutes with push-forwards

$$
\psi_{*}\left(\mathcal{L}_{X} Y\right)=\mathcal{L}_{\psi_{*} X} \psi_{*} Y
$$

and pull-backs

$$
\psi^{*}\left(\mathcal{L}_{\psi_{*} X} \alpha\right)=\mathcal{L}_{X} \psi^{*} \alpha
$$

where $\psi: \mathcal{M} \rightarrow \mathcal{N}$ is a smooth one-to-one map between differential manifolds $\mathcal{M}$ and $\mathcal{N}, X, Y \in \Gamma T \mathcal{M}$ and $\alpha \in \Gamma \Lambda \mathcal{N}$.

\subsubsection{Example}

Let $\{x, y\}$ be the coordinates of a chart $(\mathcal{U}, \phi)$ on a 2-dimensional differential manifold $\mathcal{M}$. Then

$$
\begin{aligned}
\mathcal{L}_{\cos (y) \partial_{x}}[\sin (x) d y] & =\mathcal{L}_{\cos (y) \partial_{x}}[\sin (x)] d y+\sin (x) \mathcal{L}_{\cos (y) \partial_{x}} d y \\
& =\cos (y) \partial_{x}[\sin (x)] d y+\sin (x) d\left(\mathcal{L}_{\cos (y) \partial_{x}} y\right) \\
& =\cos (y) \cos (x) d y+\sin (x) d\left[\cos (y) \partial_{x} y\right] \\
& =\cos (y) \cos (x) d y
\end{aligned}
$$


and

$$
\begin{aligned}
& \mathcal{L}_{\cos (y) \partial_{x}}\left[\sin (x) \partial_{y}\right]=\mathcal{L}_{\cos (y) \partial_{x}}[\sin (x)] \partial_{y}+\sin (x) \mathcal{L}_{\cos (y) \partial_{x}} \partial_{y} \\
& =\cos (y) \partial_{x}[\sin (x)] \partial_{y}-\sin (x) \mathcal{L}_{\partial_{y}}\left[\cos (y) \partial_{x}\right] \\
& =\cos (y) \cos (x) \partial_{y}-\sin (x) \mathcal{L}_{\partial_{y}}[\cos (y)] \partial_{x}-\sin (x) \cos (y) \mathcal{L}_{\partial_{y}} \partial_{x} \\
& =\cos (y) \cos (x) \partial_{y}-\sin (x) \partial_{y}[\cos (y)] \partial_{x} \\
& =\cos (y) \cos (x) \partial_{y}+\sin (x) \sin (y) \partial_{x} \text {. }
\end{aligned}
$$

\subsection{Linear connections on tensor bundles}

So far we have introduced two important differential operators : the exterior derivative $d$ that acts on differential forms and the Lie derivative that acts on any tensor field. However, we do not as yet have anything that resembles a "directional derivative" of tensors along vector fields. For example, one often constructs the conventional vector field $(\boldsymbol{u} \cdot \nabla) \boldsymbol{v}$ on $\mathbb{R}^{3}$ out of two conventional vector fields $\boldsymbol{u}$ and $\boldsymbol{v}$ where

$$
\begin{aligned}
& \boldsymbol{v}=a \boldsymbol{i}+b \boldsymbol{j}+c \boldsymbol{k}, \\
& (\boldsymbol{u} \cdot \nabla) \boldsymbol{v} \equiv[\boldsymbol{u} \cdot \operatorname{grad}(a)] \boldsymbol{i}+[\boldsymbol{u} \cdot \operatorname{grad}(b)] \boldsymbol{j}+[\boldsymbol{u} \cdot \operatorname{grad}(a)] \boldsymbol{k} .
\end{aligned}
$$

Let us define the operator $\mathcal{D}$ where $\mathcal{D}(\boldsymbol{u}, \boldsymbol{v}) \equiv(\boldsymbol{u} \cdot \nabla) \boldsymbol{v}$ and $\mathcal{D}(\boldsymbol{u}, f) \equiv$ $\boldsymbol{u} \cdot \operatorname{grad}(f)$ where $f$ is a scalar on $\mathbb{R}^{3}$. Note that $\mathcal{D}(f \boldsymbol{u}, \boldsymbol{v})=f \mathcal{D}(\boldsymbol{u}, \boldsymbol{v})$ i.e. $\mathcal{D}$ is linear in its first argument. Furthermore it obeys the Leibniz rule on its second argument i.e. $\mathcal{D}(\boldsymbol{u}, f \boldsymbol{v})=\mathcal{D}(\boldsymbol{u}, f) \boldsymbol{v}+f \mathcal{D}(\boldsymbol{u}, \boldsymbol{v})$. We already have a differential operator, the Lie derivative, that maps two vector fields on $\mathcal{M}$ to another vector field on $\mathcal{M}$. However, although it obeys the Leibnitz rule

$$
\mathcal{L}_{X}(f Y)=\left(\mathcal{L}_{X} f\right) Y+f \mathcal{L}_{X} Y
$$

it is not linear in its first argument since

$$
\begin{aligned}
\mathcal{L}_{f X} Y & =-\mathcal{L}_{Y}(f X) \\
& =-\left(\mathcal{L}_{Y} f\right) X+f \mathcal{L}_{X} Y .
\end{aligned}
$$


So, in order to discuss "directional derivatives" of tensor fields on differential manifolds we need to introduce some new machinery.

A linear connection $\nabla$ on a differential manifold $\mathcal{M}$ is a map

$$
\begin{aligned}
\nabla: \Gamma T \mathcal{M} \times \Gamma T \mathcal{M} & \rightarrow \Gamma T \mathcal{M} \\
(X, Y) & \rightarrow \nabla_{X} Y
\end{aligned}
$$

such that

$$
\begin{aligned}
& \nabla_{X}(f Y)=\left(\nabla_{X} f\right) Y+f \nabla_{X} Y, \\
& \nabla_{X} f=X f \\
& \nabla_{f X} Y=f \nabla_{X} Y
\end{aligned}
$$

where $f \in \mathcal{F}(\mathcal{M})$ and is extended to arbitrary type tensor fields by demanding that it commutes with contractions

$$
\nabla_{X}[\alpha(Y)]=\left(\nabla_{X} \alpha\right)(Y)+\alpha\left(\nabla_{X} Y\right),
$$

where $\alpha \in \Gamma \Lambda_{1} \mathcal{M}$, and is a tensor derivation

$$
\nabla_{X}(S \otimes T)=\nabla_{X} S \otimes T+S \otimes \nabla_{X} T
$$

where $S$ and $T$ are tensors on $\mathcal{M}$. Unlike the Lie derivative a linear connection is, by definition, linear in its first argument. Furthermore, the above properties are not enough to specify $\nabla$ uniquely. There are many maps that satisfy the defining properties of a linear connection.

\subsubsection{Example}

Let $(\mathcal{U}, \phi)$ be a chart with coordinates $\{x, y\}$ on a 2-dimensional differential manifold $\mathcal{M}$. A linear connection is fixed by giving its action 
on $\left\{\partial_{x}, \partial_{y}\right\}$, say

$$
\begin{aligned}
& \nabla_{\partial_{x}} \partial_{x}=x \partial_{y}, \\
& \nabla_{\partial_{x}} \partial_{y}=0, \\
& \nabla_{\partial_{y}} \partial_{x}=y^{2} \partial_{x}, \\
& \nabla_{\partial_{y}} \partial_{y}=\sin (x y) \partial_{y},
\end{aligned}
$$

and inducing its action on all other vector fields and arbitrary type tensors by the fundamental properties of all linear connections. For example, on the co-frame $\{d x, d y\}$ dual to $\left\{\partial_{x}, \partial_{y}\right\}$

$$
\begin{aligned}
& \left(\nabla_{\partial_{x}} d y\right)\left(\partial_{x}\right)=\partial_{x}\left[d y\left(\partial_{x}\right)\right]-d y\left(\nabla_{\partial_{x}} \partial_{x}\right)=-x \\
& \left(\nabla_{\partial_{x}} d y\right)\left(\partial_{y}\right)=\partial_{x}\left[d y\left(\partial_{y}\right)\right]-d y\left(\nabla_{\partial_{x}} \partial_{y}\right)=0
\end{aligned}
$$

SO

$$
\nabla_{\partial_{x}} d y=-x d x
$$

Furthermore

$$
\begin{aligned}
\nabla_{\partial_{x}}[\cos (x) d y] & =\partial_{x}[\cos (x)] d y+\cos (x) \nabla_{\partial_{x}} d y \\
& =-\sin (x) d y-\cos (x) x d x
\end{aligned}
$$

\subsubsection{Connection 1-forms}

Every linear connection $\nabla$ on an $n$-dimensional differential manifold $\mathcal{M}$ has a set of $n^{2}$ differential 1-forms $\left\{\omega_{b}^{a}\right\}$, known as connection 1-forms, associated with each basis $\left\{X_{a}\right\}$ for $\Gamma T \mathcal{M}$. They are given by

$$
\nabla_{X_{a}} X_{b}=\omega_{b}^{c}\left(X_{a}\right) X_{c} .
$$

The fundamental properties of $\nabla$ induce its action on the co-frame $\left\{e^{a}\right\}$ dual to $\left\{X_{a}\right\}$ :

$$
\begin{aligned}
\left(\nabla_{X_{a}} e^{b}\right)\left(X_{c}\right) & =\nabla_{X_{a}}\left[e^{b}\left(X_{c}\right)\right]-e^{b}\left(\nabla_{X_{a}} X_{c}\right) \\
& =-e^{b}\left(\nabla_{X_{a}} X_{c}\right)
\end{aligned}
$$


and so

$$
\nabla_{X_{a}} e^{b}=-\omega_{c}^{b}\left(X_{a}\right) e^{c}
$$

Connection 1-forms induced by different bases are related by

$$
\omega_{b}^{\prime a}=\Lambda_{b}{ }^{c} \omega_{c}^{e}{ }_{c}^{-1} e^{a}+\Lambda_{c}^{-1} d \Lambda_{b}^{c}
$$

where

$$
\begin{aligned}
& X_{a}^{\prime}=\Lambda_{a}^{b} X_{b}, \\
& X_{a}=\Lambda_{a}^{-1}{ }^{b} X_{b}^{\prime}, \\
& \nabla_{X_{a}^{\prime}} X_{b}^{\prime}=\omega^{\prime c}{ }_{b}\left(X_{a}^{\prime}\right) X_{c}^{\prime} .
\end{aligned}
$$

\subsubsection{Torsion}

Any linear connection and the Lie derivative can be combined to form two important tensor fields called torsion and curvature. The torsion operator $\mathcal{T}: \Gamma T \mathcal{M} \times \Gamma T \mathcal{M} \rightarrow \Gamma T \mathcal{M}$ induced by a linear connection $\nabla$ on $\mathcal{M}$ is

$$
\mathcal{T}_{X, Y} \equiv \nabla_{X} Y-\nabla_{Y} X-[X, Y],
$$

where $X, Y \in \Gamma T \mathcal{M}$. Since $\mathcal{T}$ can be shown to be linear in all arguments,

$$
\begin{aligned}
& \mathcal{T}_{X+Y, Z}=\mathcal{T}_{X, Z}+\mathcal{T}_{Y, Z}, \\
& \mathcal{T}_{X, Y+Z}=\mathcal{T}_{X, Y}+\mathcal{T}_{X, Z}, \\
& \mathcal{T}_{f X, Y}=\mathcal{T}_{X, f Y}=f \mathcal{T}_{X, Y},
\end{aligned}
$$

where $f \in \mathcal{F}(\mathcal{M})$ and $Z \in \Gamma T \mathcal{M}$, there must exist a type $(2,1)$ tensor field $T$ on $\mathcal{M}$, called the torsion of $\nabla$, given by

$$
\alpha\left(\mathcal{T}_{X, Y}\right)=T(X, Y, \alpha)
$$


where $\alpha \in \Gamma \Lambda_{1} \mathcal{M}$. Given any frame $\left\{X_{a}\right\}$ and dual co-frame $\left\{e^{a}\right\}$ on $\mathcal{M}$ one can construct a set of $n=\operatorname{dim} \mathcal{M}$ torsion 2-forms $\left\{T^{a}\right\}$ where

$$
T=2 T^{a} \otimes X_{a}
$$

and which, in terms of the connection 1-forms $\left\{\omega^{a}{ }_{b}\right\}$ associated with $\left\{X_{a}\right\}$ and its dual $\left\{e^{a}\right\}$, can be shown to be

$$
T^{a}=d e^{a}+\omega^{a}{ }_{b} \wedge e^{b} .
$$

Equation (65) is known as Cartan's first structure equation. It can be shown that $d$ and $\nabla$ are related by

$$
d \alpha=e^{a} \wedge \nabla_{X_{a}} \alpha+T^{a} \wedge \iota_{X_{a}} \alpha
$$

where $\alpha \in \Gamma \Lambda \mathcal{M}$. A straightforward proof of (66) involves induction and begins by using (65) and (61) to verify (66) on 0 -forms and 1-forms. That (66) holds on arbitrary degree differential forms then follows by assuming that it holds on $(p-1)$-forms and using the properties of $d$ and $\nabla$ to show that it holds on $p$-forms.

A linear connection with vanishing torsion, i.e. $T=0$, is said to be torsion-free.

\subsubsection{Curvature}

Another object induced by a linear connection $\nabla$ on $\mathcal{M}$ is the curvature operator $\mathcal{R}: \Gamma T \mathcal{M} \times \Gamma T \mathcal{M} \times \Gamma T \mathcal{M} \rightarrow \Gamma T \mathcal{M}$

$$
\mathcal{R}_{X, Y} Z \equiv \nabla_{X} \nabla_{Y} Z-\nabla_{Y} \nabla_{X} Z-\nabla_{[X, Y]} Z
$$

where $X, Y, Z \in \Gamma T \mathcal{M}$. Like the torsion operator it can be shown to be linear in all arguments. Therefore there exists a type $(3,1)$ tensor field $R$ called the curvature tensor of $\nabla$ :

$$
R(X, Y, Z, \alpha)=\alpha\left(\mathcal{R}_{X, Y} Z\right) .
$$


Associated with any frame $\left\{X_{a}\right\}$ and dual co-frame $\left\{e^{a}\right\}$ are $n^{2}$ curvature 2-forms $\left\{R_{b}^{a}\right\}$ given by

$$
R=2 R_{b}^{a} \otimes e^{a} \otimes X_{b}
$$

which are related to the connection 1-forms $\left\{\omega^{a}{ }_{b}\right\}$ by Cartan's second structure equation

$$
R_{b}^{a}=d \omega_{b}^{a}+\omega_{c}^{a} \wedge \omega_{b}^{c} .
$$

If $\mathcal{M}$ is equipped with a metric then one can construct the curvature scalar $\mathcal{R} \in \mathcal{F}(\mathcal{M})$

$$
\mathcal{R} \equiv \iota_{X^{b} \iota_{X_{a}}} R_{b}^{a}
$$

where

$$
X^{a}=\tilde{e}^{a} .
$$

\subsubsection{The Bianchi identities}

The power and elegance of exterior differential calculus over general tensor calculus is very clearly demonstrated when deriving the Bianchi identities. These are canonical relationships that must be satisfied by the curvature and torsion of any linear connection and are consequences of (65) and (69). Taking the exterior derivative of (65) and using $d^{2}=0$ yields

$$
\begin{aligned}
d T^{a} & =d^{2} e^{a}+d \omega^{a}{ }_{b} \wedge e^{b}-\omega^{a}{ }_{b} \wedge d e^{b} \\
& =d \omega^{a}{ }_{b} \wedge e^{b}-\omega^{a}{ }_{b} \wedge\left(T^{b}-\omega^{b}{ }_{c} \wedge e^{c}\right) \\
& =-\omega^{a}{ }_{b} \wedge T^{b}+\left(d \omega^{a}{ }_{b}+\omega^{a}{ }_{c} \wedge \omega^{c}{ }_{b}\right) \wedge e^{b} .
\end{aligned}
$$

Thus, using (69) we obtain Bianchi's first identity

$$
d T^{a}+\omega_{b}^{a} \wedge T^{b}=R_{b}^{a} \wedge e^{b} .
$$


Taking the exterior derivative of (69) leads to

$$
\begin{aligned}
d R_{b}^{a} & =d^{2} \omega_{b}^{a}+d \omega^{a}{ }_{c} \wedge \omega^{c}{ }_{b}-\omega^{a}{ }_{c} \wedge d \omega^{c}{ }_{b} \\
& =\left(R^{a}{ }_{c}-\omega^{a}{ }_{d} \wedge \omega^{d}{ }_{c}\right) \wedge \omega^{c}{ }_{b}-\omega^{a}{ }_{c} \wedge\left(R^{c}{ }_{b}-\omega^{c}{ }_{d} \wedge \omega_{b}{ }_{b}\right)
\end{aligned}
$$

or

$$
d R_{b}^{a}+\omega_{c}^{a} \wedge R_{b}^{c}-\omega_{b}^{c} \wedge R_{c}^{a}=0
$$

which is Bianchi's second identity. We could have derived these identities from the fundamental definitions of torsion and curvature, equations (63) and (67), by acting with $\nabla$ on them and judiciously antisymmetrizing with respect to the various vector arguments. However, the computations are considerably more complicated and nowhere near as transparent as those just given.

\subsubsection{Non-metricity}

Let $\mathcal{M}$ be a differential manifold with metric tensor $g$ and linear connection $\nabla$. The non-metricity $Q$ of $\nabla$ is the type $(3,0)$ tensor field

$$
Q(X, Y, Z) \equiv\left(\nabla_{X} g\right)(Y, Z)
$$

where $X, Y, Z \in \Gamma T \mathcal{M}$ and if $Q=0$ the linear connection is said to be metric-compatible. It can be shown that the triple $(g, T, Q)$ determines $\nabla$ i.e. a linear connection is completely specified in terms of the metric, torsion and non-metricity tensor fields. If $\nabla$ is metric-compatible then $\nabla_{X_{a}}\left[g\left(X_{b}, X_{c}\right)\right]=0$ if $\left\{X_{a}\right\}$ is a frame where $\left\{g_{a b}=g\left(X_{a}, X_{b}\right)\right\}$ are constant, for example if $\left\{X_{a}\right\}$ is orthonormal (see section 2.4.1). Then

$$
\begin{aligned}
g\left(\nabla_{X_{a}} X_{b}, X_{c}\right)+g\left(X_{b}, \nabla_{X_{a}} X_{c}\right) & =\omega_{b}^{d}\left(X_{a}\right) g_{d c}+\omega^{d}{ }_{c}\left(X_{a}\right) g_{b d} \\
& =\omega_{c b}\left(X_{a}\right)+\omega_{b c}\left(X_{a}\right),
\end{aligned}
$$

where $\omega_{a b}=g_{a c} \omega^{c}{ }_{b}$, and so

$$
\omega_{a b}=-\omega_{b a}
$$

if $\nabla$ is metric-compatible and $\left\{g\left(X_{a}, X_{b}\right)\right\}$ are constant. 


\subsubsection{Covariant exterior derivatives}

A cursory examination of Cartan's first structure equation (65) and the Bianchi identities (71) and (72) suggests the introduction of an exterior differential operator on the space of certain index-carrying differential forms such as $R^{a}{ }_{b}$ and $T^{a}$. Technically we want an exterior differential operator on forms that take values in the bundle of linear frames. Let $\Omega_{\mathcal{M}}(A)$ denote the set of index-carrying differential forms obtained by contracting the tensor $A$, on $\mathcal{M}$, with all frames $\left\{X_{a}\right\}$ and their duals in all possible combinations (including no contractions) that yields a differential form. For example the set $\Omega_{\mathcal{M}}(\alpha), \alpha \in \Gamma \Lambda_{2} \mathcal{M}$, contains $\alpha$ itself, the indexed 1-forms $\left\{\alpha_{a}=\iota_{X_{a}} \alpha\right\}$ and the indexed scalars $\left\{\alpha_{a b}=\iota_{X_{b}} \iota_{X_{a}} \alpha\right\}$. It also contains $\left\{\alpha_{a}^{\prime}=\iota_{X_{a}^{\prime}} \alpha\right\}$ and $\left\{\alpha_{a b}^{\prime}=\iota_{X_{b}^{\prime}} \iota_{X_{a}^{\prime}} \alpha\right\}$ where $\left\{X_{a}^{\prime}\right\}$ is a different frame to $\left\{X_{a}\right\}$. The curvature $R^{a}{ }_{b}$ and torsion $T^{a}$ 2-forms are elements of $\Omega_{\mathcal{M}}(R)$ and $\Omega_{\mathcal{M}}(T)$ respectively (see equations (64) and (68)). Furthermore, any co-frame 1-form $e^{a}$ is an element of $\Omega_{\mathcal{M}}(\mathrm{id})$ where id $=e^{a} \otimes X_{a}$. However, $\omega^{a}{ }_{b} \otimes e^{b} \otimes X_{a} \neq$ $\omega^{\prime a}{ }_{b} \otimes e^{\prime b} \otimes X_{a}^{\prime}$ in general (see $\left.(62)\right)$ and so a type $(2,1)$ tensor $S$ such that $\omega^{a}{ }_{b}=S\left(X_{b}, e^{a},-\right)$ and $\omega^{\prime a}{ }_{b}=S\left(X_{b}^{\prime}, e^{\prime a},-\right)$ for all $\left\{X_{a}\right\} \neq$ $\left\{X_{a}^{\prime}\right\}$ does not exist. Put another way, the connection 1-forms do not transform homogenously under a change of frame.

The covariant exterior derivative $D$ is a map between the spaces $\Omega_{\mathcal{M}}(\cdot)$. It follows a similar pattern to the coordinate-based covariant derivative, often denoted by ; in the literature,

$$
\begin{aligned}
D A^{a \ldots b}{ }_{c \ldots d} \equiv & d A^{a \ldots b_{c \ldots d}}+\omega^{a}{ }_{e} \wedge A^{e \ldots b}{ }_{c \ldots d}+\cdots+\omega^{b}{ }_{e} \wedge A^{a \ldots e}{ }_{c \ldots d}{ }_{c \ldots d} \\
& -\omega^{e}{ }_{c} \wedge A^{a \ldots{ }_{e} \ldots d}-\cdots-\omega^{e}{ }_{d} \wedge A^{a \ldots b}{ }_{c \ldots e},
\end{aligned}
$$

where $A^{a \ldots b}{ }_{c \ldots d} \in \Omega_{\mathcal{M}}(A)$ and $B^{a \ldots b}{ }_{c \ldots d} \in \Omega_{\mathcal{M}}(B)$ are frame-valued differential forms whose indices are induced by $\left\{X_{a}\right\}$ and $\left\{\omega^{a}{ }_{b}\right\}$ are the connection 1-forms with respect to $\left\{X_{a}\right\}$. The $\ldots$ indicates omitted indices and terms that follow the same pattern as those shown. For 
example

$$
\begin{aligned}
& D e^{a}=d e^{a}+\omega_{b}^{a} \wedge e^{b}=T^{a}, \\
& D R_{b}^{a}=d R_{b}^{a}+\omega^{a}{ }_{c} \wedge R_{b}^{c}-\omega^{c}{ }_{b} \wedge R_{c}^{a}=0, \\
& D T^{a}=d T^{a}+\omega^{a}{ }_{b} \wedge T^{b}=R^{a}{ }_{b} \wedge e^{b} .
\end{aligned}
$$

By inspecting (75) it can be shown that

$D\left(A^{a \ldots \ldots}{ }_{c \ldots d} \wedge B^{e \ldots f}{ }_{g \ldots h}\right)=D A^{a \ldots b}{ }_{c \ldots d} \wedge B^{e \ldots f}{ }_{g \ldots h}+(-1)^{p} A^{a \ldots b}{ }_{c \ldots d} \wedge D B^{e \ldots f}{ }_{g \ldots h}$

where $A^{a \ldots b}{ }_{c \ldots d} \in \Omega_{\mathcal{M}}(A)$ is a $p$-form and $B^{a \ldots b}{ }_{c \ldots d} \in \Omega_{\mathcal{M}}(B)$.

If $\mathcal{M}$ possesses a metric $g$ and $\nabla$ is metric-compatible then

$$
D g_{a b}=0
$$

and indices can be "lowered" and "raised" through $D$ with respect to the metric components $g_{a b}=g\left(X_{a}, X_{b}\right)$ and $g^{a b}=g^{-1}\left(e^{a}, e^{b}\right)$, e.g.

$$
D R_{a b}=D\left(g_{a c} R_{b}^{c}\right)=g_{a c} D R_{b}^{c}
$$

\subsubsection{Covariant derivatives, parallel transport and autopar- allels}

Let $\nabla$ be a linear connection on an $n$-dimensional differential manifold $\mathcal{M}$. Let $C$ be a curve in $\mathcal{M}$ parametrized by $\tau$ i.e.

$$
\begin{aligned}
C:[0,1] & \rightarrow \mathcal{M} \\
\tau & \rightarrow p=C(\tau)
\end{aligned}
$$

and denote $\dot{C} \equiv C_{*} \partial_{\tau}$. The covariant derivative of a vector field $X \in \Gamma T \mathcal{M}$ along $C$ is the vector field $\nabla_{\dot{C}} X$ and if

$$
\nabla_{\dot{C}} X=0
$$


then $X$ is said to be parallel along $C$. With respect to a chart $(\mathcal{U}, \phi)$ with coordinates $\left\{x^{a}\right\}$ equation (76) has the form

$$
\begin{aligned}
\nabla_{\dot{C}} X & =\nabla_{\dot{C}}\left(\xi^{a} \partial_{a}\right) \\
& =\left(\dot{C} \xi^{a}\right) \partial_{a}+\xi^{a} \nabla_{\dot{C}} \partial_{a} \\
& =\left(\dot{C} \xi^{a}\right) \partial_{a}+\xi^{a} \omega^{b}{ }_{a}(\dot{C}) \partial_{b} \\
& =\left[\dot{C} \xi^{a}+\xi^{b} \omega^{a}{ }_{b}(\dot{C})\right] \partial_{a} \\
& =0
\end{aligned}
$$

where $\left\{\xi^{a}=d x^{a}(X)\right\}$ are the components of $X$ with respect to $(U, \phi)$ and $\left\{\omega^{a}{ }_{b}\right\}$ are the connection 1-forms of $\nabla$ associated with $\left\{\partial_{a}=\right.$ $\left.\partial / \partial x^{a}\right\}$. As an ordinary differential equation

$$
\begin{aligned}
C^{*}\left[\dot{C} \xi^{a}+\xi^{b} \omega_{b}^{a}(\dot{C})\right] & =C^{*}\left[\left(C_{*} \partial_{\tau}\right) \xi^{a}+\xi^{b} \omega^{a}{ }_{b}\left(C_{*} \partial_{\tau}\right)\right] \\
& =\frac{d\left(\xi^{a} \circ C\right)}{d \tau}+\left(\xi^{b} \circ C\right)\left(C^{*} \omega^{a}{ }_{b}\right)\left(\partial_{\tau}\right) \\
& =\frac{d\left(\xi^{a} \circ C\right)}{d \tau}+\left(\xi^{b} \circ C\right)\left(\Gamma_{b c}^{a} \circ C\right) \frac{d C^{c}}{d \tau}=0
\end{aligned}
$$

where $\left\{\Gamma_{b c}^{a}=\omega^{a}{ }_{b}\left(\partial_{c}\right)\right\}$. One can turn the argument around and solve the well-posed initial value problem

$$
\begin{aligned}
& \frac{d \kappa^{a}}{d \tau}+\left(\Gamma_{b c}^{a} \circ C\right) \frac{d C^{c}}{d \tau} \kappa^{b}=0, \\
& \kappa^{a}(0)=\kappa_{0}^{a},
\end{aligned}
$$

where $\left(\kappa_{0}^{1}, \ldots, \kappa_{0}^{n}\right) \in \mathbb{R}^{n}$, to obtain a vector field $Y$ attached to $C$ (i.e. defined only on the image of $C$ rather than the whole of $\mathcal{M}$ )

$$
Y=\left(\kappa^{a} \circ \stackrel{-1}{C}\right) \partial_{a}
$$

that is parallel along $C$. Clearly, (77) means that a choice of linear connection establishes a map between the tangent spaces of $\mathcal{M}$, i.e. it 
connects vectors at different points in $\mathcal{M}$. An autoparallel of $\nabla$ is a curve $C$ that is a solution to

$$
\nabla_{\dot{C}} \dot{C}=0
$$

i.e. an autoparallel of a linear connection is a curve whose tangent is parallel along it. As a differential equation (79) reads

$$
\frac{d^{2} C^{a}}{d \tau^{2}}+\left(\Gamma_{b c}^{a} \circ C\right) \frac{d C^{b}}{d \tau} \frac{d C^{c}}{d \tau}=0 .
$$

\subsubsection{The Levi-Civita connection}

We now have a class of potential candidates for the conventional directional derivative $(\boldsymbol{u} \cdot \nabla) \boldsymbol{v}$ on $\mathbb{R}^{3}$. Which linear connection should we choose? As mentioned before, a linear connection is completely specified in terms of metric, torsion and non-metricity tensors. We have already commented that $\mathbb{R}^{3}$ as a differential manifold possesses a natural global chart $\left(\mathbb{R}^{3}, \phi\right)$ with coordinates $\{x, y, z\}$ and that, for the purposes of vector analysis, we endow it with the metric

$$
g=d x \otimes d x+d y \otimes d y+d z \otimes d z .
$$

Thus, only the non-metricity and torsion remain to be specified. The unique torsion-free metric-compatible linear connection on a differential manifold with a metric is known as the Levi-Civita connection. It is this special connection that coincides with the conventional directional derivative on $\mathbb{R}^{3}$. Thus, the Levi-Civita connection on a differential manifold $(\mathcal{M}, g)$ satisfies

$$
\begin{aligned}
& \nabla_{X}[g(Y, Z)]=g\left(\nabla_{X} Y, Z\right)+g\left(Y, \nabla_{X} Z\right), \\
& \nabla_{X} Y-\nabla_{Y} X-[X, Y]=0
\end{aligned}
$$

for any $X, Y, Z \in \Gamma T \mathcal{M}$. Autoparallels of the Levi-Civita connection are called geodesics. 
The vector field $\nabla_{X} X, X \in \Gamma T \mathcal{M}$, has a nice expression in terms of exterior calculus. Let $A_{X}$ be the differential operator

$$
A_{X} \equiv \nabla_{X}-\mathcal{L}_{X}
$$

and note that $A_{X} f=0$ for $f \in \mathcal{F}(\mathcal{M})$. Hence

$$
\begin{aligned}
A_{X}[\tilde{X}(Y)] & =0 \\
& =\left(A_{X} \tilde{X}\right)(Y)+\tilde{X}\left(A_{X} Y\right) \\
& =\left(A_{X} \tilde{X}\right)(Y)+\tilde{X}\left(\nabla_{Y} X\right) \\
& =\left(\nabla_{X} \tilde{X}\right)(Y)-\left(\mathcal{L}_{X} \tilde{X}\right)(Y)+\frac{1}{2} \nabla_{Y}[g(X, X)]
\end{aligned}
$$

where the torsion-free and metric-compatible properties of $\nabla$ have been used and so, observing (57),

$$
\begin{aligned}
\nabla_{X} \tilde{X} & =\mathcal{L}_{X} \tilde{X}-\frac{1}{2} d[g(X, X)] \\
& =\iota_{X} d \tilde{X}+\frac{1}{2} d[g(X, X)] .
\end{aligned}
$$

In applications it is often useful to have an expression for the LeviCivita connection 1-forms $\left\{\omega_{b}^{a}\right\}$ in terms of a co-frame $\left\{e^{a}\right\}$ with dual frame $\left\{X_{a}\right\}$ such that $g\left(X_{a}, X_{b}\right)$ is constant. Referring to (65) we see that

$$
d e^{a}+\omega^{a}{ }_{b} \wedge e^{b}=0
$$

since $\nabla$ is torsion-free. Acting with the interior operator on (81) yields

$$
\iota_{X_{a}} d e_{b}+\omega_{b c}\left(X_{a}\right) e^{c}-\omega_{b a}=0
$$

where $e_{a}=\tilde{X}_{a}$ and $\omega_{a b}=g_{a c} \omega^{c}{ }_{b}$ which, combined with (74), can be used to show that

$$
\omega_{a b}=\frac{1}{2}\left(\iota_{X_{b}} d e_{a}-\iota_{X_{a}} d e_{b}+e_{c} \iota_{X_{a}} \iota_{X_{b}} d e^{c}\right) .
$$


With respect to the orthonormal co-frame

$$
\begin{aligned}
& e^{1}=d x \\
& e^{2}=d y \\
& e^{3}=d z
\end{aligned}
$$

on $\mathbb{R}^{3}$ the Levi-Civita connection 1 -forms vanish, since $d d=0$, as do the curvature 2 -forms. Manifolds with Levi-Civita connections whose curvature vanishes are said to be flat.

Old-fashioned coordinate-based methods of calculating curvature tend to employ the Christoffel symbols, which are the components $\Gamma_{b c}^{a}$ of the Levi-Civita connection 1-forms based on a coordinate frame $\left\{\partial_{a}\right\}$. In general it is computationally advantageous to use LeviCivita connection 1-forms based on an orthonormal frame. Since $\omega_{a b}=-\omega_{b a}$ with respect to an orthonormal frame $\left\{X_{a}\right\}$ at most $n(n-1) / 2(n=\operatorname{dim} \mathcal{M})$ calculations must be made to obtain $\left\{\omega_{a b}\right\}$. In general $n^{2}(n+1) / 2$ calculations must be made to obtain the Christoffel symbols.

\subsubsection{Example : differential geometry on the 2-sphere}

Let us consider the differential manifold $\left(S_{2}, g\right)$. The metric on $S_{2}$ can be written locally

$$
g=d \theta \otimes d \theta+\sin ^{2}(\theta) d \varphi \otimes d \varphi
$$

where $0<\theta<\pi$ and $0<\varphi<2 \pi$ are the ranges of the coordinates of a chart $(\mathcal{U}, \phi)$ where $\mathcal{U}$ is $S_{2}$ excluding a longitudinal line that joins the two poles i.e. the limit points $\theta \rightarrow 0$ and $\theta \rightarrow \pi$. An orthonormal co-frame on $\mathcal{U} \subset S_{2}$ is

$$
\begin{aligned}
e^{1} & =d \theta \\
e^{2} & =\sin (\theta) d \varphi
\end{aligned}
$$


and has the dual orthonormal frame

$$
\begin{aligned}
& X_{1}=\frac{\partial}{\partial \theta}, \\
& X_{2}=\frac{1}{\sin (\theta)} \frac{\partial}{\partial \varphi} .
\end{aligned}
$$

Thus

$$
\begin{aligned}
& d e^{1}=d^{2} \theta=0, \\
& d e^{2}=d[\sin (\theta)] \wedge d \varphi+\sin (\theta) d^{2} \varphi=\cos (\theta) d \theta \wedge d \varphi=\tan (\theta) e^{1} \wedge e^{2}
\end{aligned}
$$

and so the Levi-Civita connection 1-form $\omega_{12}$ with respect to $\left\{X_{1}, X_{2}\right\}$ is

$$
\begin{aligned}
\omega_{12} & =\frac{1}{2}\left(\iota_{X_{2}} d e_{1}-\iota_{X_{1}} d e_{2}+e_{a} \iota_{X_{1}} \iota_{X_{2}} d e^{a}\right) \\
& =-\tan (\theta) e^{2} \\
& =-\cos (\theta) d \varphi
\end{aligned}
$$

where $e_{1}=e^{1}$ and $e_{2}=e^{2}$ has been used which follows because $g\left(X_{a}, X_{b}\right)=\delta_{a b}$. The other three Levi-Civita connection 1-forms are

$$
\begin{aligned}
& \omega_{21}=-\omega_{12}=\cos (\theta) d \varphi \\
& \omega_{11}=\omega_{22}=0 .
\end{aligned}
$$

The curvature 2-forms are

$$
\begin{aligned}
R_{12} & =d \omega_{12}+\omega_{11} \wedge \omega^{1}{ }_{2}+\omega_{12} \wedge \omega^{2}{ }_{2}, \\
& =d \omega_{12}, \\
& =\sin (\theta) d \theta \wedge d \varphi, \\
& =e^{1} \wedge e^{2}
\end{aligned}
$$


and

$$
\begin{aligned}
& R_{21}=-R_{12}=-e^{1} \wedge e^{2} \\
& R_{11}=R_{22}=0
\end{aligned}
$$

so the curvature scalar is

$$
\begin{aligned}
\mathcal{R} & =2 \iota_{X_{1}} \iota_{X_{2}} R_{21} \\
& =2 .
\end{aligned}
$$

Note that the final result is frame-independent and is valid over all of $S_{2}$ because the longitudinal line excluded from $(\mathcal{U}, \phi)$ can be chosen anywhere.

\section{Newtonian continuum mechanics}

Newtonian absolute time must be accommodated if we are to discuss Newtonian continuum mechanics on differential manifolds. One method of accomplishing this is in terms of smooth tensor-valued maps from an interval $I \subset \mathbb{R}$ into the space of tensor fields $\Gamma \mathbf{T}_{q}^{p} \mathcal{M}$,

$$
\begin{aligned}
T: I & \rightarrow \Gamma \mathbf{T}_{q}^{p} \mathcal{M} \\
t & \rightarrow T_{t},
\end{aligned}
$$

where $t$ is the Newtonian absolute time. Denote the space of all such maps by $\mathbf{T}_{\mathcal{M}}^{(q, p)}$ and note the smoothness of $T$ means that the derivative $\partial_{t} T$ of $T \in \mathbf{T}_{\mathcal{M}}^{(q, p)}$ with respect to $t$

$$
\partial_{t} T_{t} \equiv \lim _{\varepsilon \rightarrow 0} \frac{1}{\varepsilon}\left(T_{t+\varepsilon}-T_{t}\right)
$$

is also an element of $\mathbf{T}_{\mathcal{M}}^{(q, p)}$ as are all higher order derivatives of $T$ with respect to $t$. Operations defined on sections of the tensor bundle $\mathbf{T}_{q}^{p} \mathcal{M}$ 
naturally induce operations on the elements of $\mathbf{T}_{\mathcal{M}}^{(q, p)}$. For example

$$
\left(\mathcal{L}_{X} Y\right)_{t}=\mathcal{L}_{X_{t}} Y_{t}
$$

where $X, Y \in T_{\mathcal{M}} \equiv \mathbf{T}_{\mathcal{M}}^{(0,1)}$ and so $X_{t}, Y_{t} \in \Gamma \mathbf{T}_{0}^{1} \mathcal{M}=\Gamma T \mathcal{M}$. For simplicity, although it is a slight abuse of language, we will refer to the elements of $\mathbf{T}_{\mathcal{M}}^{(q, p)}$ as type $(q, p)$ tensor fields and elements of $T_{\mathcal{M}}$ as vector fields on $\mathcal{M}$. Note that any bona fide tensor field $S$ given on $\mathcal{M}$, i.e. $S \in \Gamma \mathbf{T}_{q}^{p} \mathcal{M}$, corresponds to the element $T \in \mathbf{T}_{\mathcal{M}}^{(q, p)}$ given by $\partial_{t} T=$ 0 and $T_{t}=S$. We will use the same symbol for corresponding elements of $\Gamma \mathbf{T}_{q}^{p} \mathcal{M}$ and $\mathbf{T}_{\mathcal{M}}^{(q, p)}$. Similarly, let $\Lambda_{\mathcal{M}}^{p}$ be the space of smooth maps from $I$ into $\Gamma \Lambda_{p} \mathcal{M}$ and denote $\mathcal{F}_{\mathcal{M}}=\Lambda_{\mathcal{M}}^{0}$. Again, we will call elements of $\Lambda_{\mathcal{M}}^{p}$ differential $p$-forms on $\mathcal{M}$ and elements of $\mathcal{F}_{\mathcal{M}}$ scalar fields on $\mathcal{M}$ and use the same symbol for elements in $\Lambda_{\mathcal{M}}^{p}$ corresponding to $\Gamma \Lambda_{p} \mathcal{M}$ and those in $\mathcal{F}_{\mathcal{M}}$ corresponding to $\mathcal{F}(\mathcal{M})$. Note that $\partial_{t}$ and $d$ commute on differential forms in $\Lambda_{\mathcal{M}}^{p}$ i.e. $\partial_{t} d \alpha=d \partial_{t} \alpha$ for $\alpha \in \Lambda_{\mathcal{M}}^{p}$. In the same way as with tensor-valued maps we introduce the space of smooth $p$-chain-valued maps $\mathcal{C}_{\mathcal{M}}^{p}$. Thus, if $c \in \mathcal{C}_{\mathcal{M}}^{p}$ then $c_{t}$ is, for each $t \in I$, a $p$-chain on $\mathcal{M}$.

In the Euler picture a continuous body can be modelled by a manifold $\mathcal{B} \subset \mathbb{R}^{3}$, $\operatorname{dim} \mathcal{B}=3$, with the standard Euclidean metric $g \in \Gamma \mathbf{T}_{2}^{0} \mathbb{R}^{3}$, a positive-definite scalar field $\rho \in \mathcal{F}_{\mathcal{B}}$ called the density, a vector field $V \in T_{\mathcal{B}}$ called the velocity and a Cauchy stress symmetric tensor $S \in \mathbf{T}_{\mathcal{B}}^{(2,0)}$ that describes its constitutive properties. A Cauchy stress 2 -form $\tau_{X} \in \Lambda_{\mathcal{M}}^{2}$ with respect to $X \in T_{\mathcal{M}}$ is

$$
\tau_{X}=\star[S(-, X)]
$$

where $\star 1$ is an orientation for $\mathcal{B}$. A vector field $K \in \Gamma T \mathbb{R}^{3}$ that satisfies

$$
\mathcal{L}_{K} g=0
$$

is known as a Killing vector. Each $K$ induces a 1-parameter family of maps $\left\{\varphi_{\lambda}\right\}$ called an isometry (a diffeomorphism from $\mathbb{R}^{3}$ to itself that 
preserves the metric). A complete set of isometries on $\mathbb{R}^{3}$ forms the 6 -dimensional group of rigid rotations and translations of $\mathbb{R}^{3}$. With respect to a global Cartesian chart with coordinates $\{x, y, z\}$

$$
g=d x \otimes d x+d y \otimes d y+d z \otimes d z
$$

a complete set of Killing vectors is

$$
\begin{aligned}
& K_{1}=\partial_{x} \\
& K_{2}=\partial_{y} \\
& K_{3}=\partial_{z} \\
& K_{4}=x \partial_{y}-y \partial_{x} \\
& K_{5}=y \partial_{z}-z \partial_{y} \\
& K_{6}=z \partial_{x}-x \partial_{z}
\end{aligned}
$$

where $\left\{K_{1}, K_{2}, K_{3}\right\}$ generate translations and $\left\{K_{4}, K_{5}, K_{6}\right\}$ generate rotations.

Cauchy's balance laws for momentum and angular momentum can be written as the single expression

$$
\frac{d}{d t} \int_{\Omega} \rho \tilde{V}(K) \star 1=\int_{\partial \Omega} \tau_{K}+\int_{\Omega} \beta_{K}
$$

where $\Omega \in \mathcal{C}_{\mathcal{B}}^{3}, K \in T_{\mathbb{R}^{3}}$ corresponds to a Killing vector $K \in \Gamma T \mathbb{R}^{3}$ and the body force 3 -form $\beta_{K} \in \Lambda_{\mathcal{B}}^{3}$ is linear in its argument, i.e. $\beta_{f X}=f \beta_{X}$ and $\beta_{X+Y}=\beta_{X}+\beta_{Y}, X, Y \in \Gamma T \mathcal{B}, f \in \mathcal{F}(\mathcal{B})$. For example, if gravity is acting on $\mathcal{B}$ and $\tilde{\mathfrak{g}} \in \Gamma T \mathcal{B}$ is the Newtonian gravitational acceleration field then $\beta_{X}=\rho \mathfrak{g}(K) \star 1$. Each Killing vector leads to a component of the conventional linear momentum or angular momentum conservation laws. If $\Omega$ is chosen so that

$$
V=\left[\left(\partial_{t} \Omega_{t}^{a}\right) \circ \stackrel{-1}{\Omega_{t}}\right] \frac{\partial}{\partial x^{a}}
$$


where $x^{a}=\Omega_{t}^{a}(p)$ are the components of $\Omega_{t}$ with respect to a chart with coordinates $\left\{x^{a}\right\}$, it can be shown that

$$
\frac{d}{d t} \int_{\Omega} \alpha=\int_{\Omega}\left(\partial_{t} \alpha+\mathcal{L}_{V} \alpha\right)
$$

where $\alpha \in \Lambda_{\mathcal{B}}^{3}$. The chain $\Omega$ is said to be co-moving with the medium. Conservation of mass is expressed as

$$
\frac{d}{d t} \int_{\Omega} \rho \star 1=0
$$

which, using (84), becomes

$$
\begin{aligned}
\int_{\Omega}\left[\partial_{t} \rho \star 1+\mathcal{L}_{V}(\rho \star 1)\right] & =\int_{\Omega}\left[\partial_{t} \rho \star 1+d \iota_{V}(\rho \star 1)\right] \\
& =\int_{\Omega}\left[\partial_{t} \rho \star 1+d(\rho \star \tilde{V})\right] \\
& =0
\end{aligned}
$$

where (57) and (27) have been used. Since this is true for any $\Omega \in \mathcal{C}_{\mathcal{B}}^{3}$ we obtain the local mass conservation law

$$
\partial_{t} \rho \star 1+d(\rho \star \tilde{V})=0 .
$$

The left-hand side of equation (83) can be written

$$
\frac{d}{d t} \int_{\Omega} \rho \tilde{V}(K) \star 1=\int_{\Omega}\left[\partial_{t} \tilde{V}(K)+\mathcal{L}_{V} \tilde{V}(K)+\tilde{V}\left(\mathcal{L}_{V} K\right)\right] \rho \star 1
$$

where (84), (86) and $\partial_{t} K=0$ have been used. However,

$$
\begin{aligned}
\tilde{V}\left(\mathcal{L}_{V} K\right) & =-\tilde{V}\left(\mathcal{L}_{K} V\right) \\
& =-\frac{1}{2} \mathcal{L}_{K}[g(V, V)] \\
& =-\frac{1}{2} K[g(V, V)]
\end{aligned}
$$


since $K$ is Killing and using (80) we see that

$$
-\frac{1}{2} K[g(V, V)]=\nabla_{V} \tilde{V}(K)-\mathcal{L}_{V} \tilde{V}(K)
$$

where $\nabla$ is the Levi-Civita connection on $\mathbb{R}^{3}$. Hence

$$
\frac{d}{d t} \int_{\Omega} \rho \tilde{V}(K) \star 1=\int_{\Omega}\left[\partial_{t} \tilde{V}(K)+\nabla_{V} \tilde{V}(K)\right] \rho \star 1
$$

and so using Stokes' theorem (38) equation (83) becomes

$$
\int_{\Omega}\left[\partial_{t} \tilde{V}(K)+\nabla_{V} \tilde{V}(K)\right] \rho \star 1=\int_{\Omega}\left(d \tau_{K}+\beta_{K}\right)
$$

which must hold for all $\Omega$. Therefore we obtain the local version of Cauchy's balance laws

$$
\rho\left[\partial_{t} \tilde{V}(K)+\nabla_{V} \tilde{V}(K)\right] \star 1=d \tau_{K}+\beta_{K} .
$$

\subsection{Example : Hydrodynamics of perfect fluids}

Let $\mathcal{B}$ be a Newtonian inviscid fluid. This means that the Cauchy stress tensor is

$$
S=-p g
$$

where $p \in \mathcal{F}_{\mathcal{B}}$ is the pressure and the density $\rho$ is a non-zero constant (the fluid is incompressible). Without loss of generality we choose $\rho=1$. Thus, $\partial_{t} \rho=0$ and $d \rho=0$ and so (86) becomes

$$
d \star \tilde{V}=0 \text {. }
$$

The volume form $\star 1$ depends only on the metric and so for each Killing vector field $K$

$$
\begin{aligned}
\mathcal{L}_{K} \star 1 & =0 \\
& =d \star \tilde{K}
\end{aligned}
$$


where (27) and (57) have been used. Therefore

$$
\begin{aligned}
d \tau_{K} & =d[-p \star \tilde{K}] \\
& =-d p \wedge \star \tilde{K} \\
& =-d p(K) \star 1 .
\end{aligned}
$$

In the absence of external body forces $\beta_{K}=0$ and Cauchy's balance laws on $\mathcal{B}$ are

$$
\partial_{t} \tilde{V}(K)+\nabla_{V} \tilde{V}(K)=-d p(K)
$$

which, since the translational Killing triad is a basis for $\Gamma T \mathbb{R}^{3}$, can be written

$$
\partial_{t} \tilde{V}+\nabla_{V} \tilde{V}=-d p .
$$

Equations (88) and (89) are Euler's equations. Probably the most useful form of (89) is obtained by applying (80) to rewrite the connection term

$$
\begin{aligned}
\nabla_{V} \tilde{V} & =\mathcal{L}_{V} \tilde{V}-\frac{1}{2} d[g(V, V)] \\
& =\iota_{V} \omega+\frac{1}{2} d[g(V, V)],
\end{aligned}
$$

where $\omega=d \tilde{V}$, to give

$$
\partial_{t} \tilde{V}+\iota_{V} \omega=-d\left[p+\frac{1}{2} g(V, V)\right] .
$$

Thus, for a steady $\left(\partial_{t} V=0\right)$ Newtonian inviscid fluid we have

$$
\iota_{V} d\left[p+\frac{1}{2} g(V, V)\right]=0
$$


since $\iota_{V} \iota_{V}=0$ i.e. the scalar $p+\frac{1}{2} g(V, V)$ is constant along integral curves of $V$. If the fluid is irrotational $(d \tilde{V}=0)$ then the Poincaré lemma tells us that on some open subset $\mathcal{U} \subset \mathcal{B}$

$$
\tilde{V}=d \varphi
$$

where $\varphi \in \mathcal{F}_{\mathcal{U}}$ is a velocity potential. Substituting (91) into (90) yields the unsteady Bernoulli equation

$$
\partial_{t} \varphi+\frac{1}{2} g(V, V)=-p+c
$$

on $\mathcal{U}$ where $c \in \mathcal{F}_{\mathcal{U}}$ satisfies $d c=0$.

Taking the exterior derivative of (90) yields

$$
\begin{aligned}
\partial_{t} \omega+d \iota_{V} \omega & =0 \\
& =\partial_{t} \omega+\mathcal{L}_{V} \omega
\end{aligned}
$$

where $d^{2}=0$ has been used. If $\Sigma \in \mathcal{C}_{\mathcal{B}}^{2}$ is a 2-chain that satisfies

$$
V=\left[\left(\partial_{t} \Sigma_{t}^{a}\right) \circ \bar{\Sigma}_{t}^{-1}\right] \frac{\partial}{\partial x^{a}}
$$

on the image of $\Sigma_{t}$, where $x^{a}=\Sigma_{t}^{a}(p)$, it can be shown that (c.f. equation (84))

$$
\frac{d}{d t} \int_{\Sigma} \beta=\int_{\Sigma}\left(\partial_{t} \beta+\mathcal{L}_{V} \beta\right)
$$

where $\beta \in \Lambda_{\mathcal{B}}^{2}$ and so

$$
\begin{aligned}
\frac{d}{d t} \int_{\Sigma} \omega & =0 \\
& =\frac{d}{d t} \int_{\Sigma} d \tilde{V} \\
& =\frac{d}{d t} \int_{\partial \Sigma} \tilde{V}
\end{aligned}
$$


The final equation indicates that the circulation $\Gamma[C]$

$$
\Gamma[C]=\int_{C} \tilde{V}
$$

around the closed 1-chain $C=\partial \Sigma \in \mathcal{C}_{\mathcal{B}}^{1}$ is conserved.

\section{Differential forms on spacetime}

Let $\mathcal{M}$ be a 4-dimensional spacetime equipped with a metric $g$ and the Levi-Civita connection $\nabla$. With respect to any orthonormal co-frame $\left\{e^{0}, e^{1}, e^{2}, e^{3}\right\}$ on $\mathcal{M}$,

$$
g=-e^{0} \otimes e^{0}+e^{1} \otimes e^{1}+e^{2} \otimes e^{2}+e^{3} \otimes e^{3} .
$$

A time-like vector field $V \in \Gamma T \mathcal{M}$ has the property $g(V, V)<0$, a space-like vector field $V$ has the property $g(V, V)>0$ and a null vector field $V$ is one such that $g(V, V)=0$. Free massive point particles are modelled on time-like geodesics and light rays are modelled on null geodesics on the spacetime manifold $\mathcal{M}$. Using a time-like vector $X_{p} \in T_{p} \mathcal{M}$ at $p \in \mathcal{M}$ one can split the time-like subset of $T_{p} \mathcal{M}$ into two equivalence classes : a future directed class of time-like vectors and a past directed class of time-like vectors. The equivalence class $\left[V_{p}\right]$ of future directed time-like vectors with representative $V_{p} \in T_{p} \mathcal{M}$ at $p \in \mathcal{M}$ is

$$
\left[V_{p}\right]=\left\{V_{p} \in T_{p} \mathcal{M} ; g\left(V_{p}, X_{p}\right)<0\right\} .
$$

Whether or not it is possible to find a time-like vector field $X \in$ $\Gamma T \mathcal{M}$ such that $\left.X\right|_{p}=X_{p}$ for all $p \in \mathcal{M}$ depends on the topology of $\mathcal{M}$. If this can be accomplished $\mathcal{M}$ is said to be time-orientable. All spacetimes considered in this article are assumed to be time-orientable. 
An observer on a spacetime $\mathcal{M}$ is a 1-chain $C$

$$
\begin{aligned}
C:[0,1] & \rightarrow \mathcal{M} \\
\tau & \rightarrow p=C(\tau)
\end{aligned}
$$

whose tangent $\dot{C}=C_{*} \partial_{\tau}$ is future directed, time-like and normalized

$$
g(\dot{C}, \dot{C})=-1
$$

i.e. $C$ is parametrized by proper time $\tau$.

\subsection{Electromagnetism}

Maxwell's equations on $\mathcal{M}$ are the pair

$$
\begin{aligned}
& d \star F=j, \\
& d F=0
\end{aligned}
$$

where $F \in \Gamma \Lambda_{2} \mathcal{M}$ is the Maxwell 2-form, $j \in \Gamma \Lambda_{3} \mathcal{M}$ is an electric current 3 -form and $\star 1 \in \Gamma \Lambda_{4} \mathcal{M}$ is an orientation on $\mathcal{M}$. A continuum of electric charge with charge density $\rho_{e} \in \mathcal{F}(\mathcal{M})$ whose constituent point particles follow integral curves of the future directed time-like normalized vector field $U \in \Gamma T \mathcal{M}$ is represented by the current $j=$ $\rho_{e} \star \tilde{U}$.

The closure of $j$

$$
d j=0
$$

follows from the first Maxwell equation since $d^{2}=0$. A space-like 3-chain $\Sigma:[0,1]^{3} \rightarrow \mathcal{M}$ is one whose normal $V_{\Sigma}$, i.e. a vector field such that

$$
\tilde{V}_{\Sigma}\left(\Sigma_{*} X\right)=0
$$


for all $X \in \Gamma T[0,1]^{3}$, is a time-like vector field attached to the image of $\Sigma$. The electric charge $Q[\Sigma]$ of $j$ across $\Sigma$ is

$$
Q[\Sigma]=\int_{\Sigma} j
$$

Let $\Omega$ be a 4-chain on $\mathcal{M}$ where $\partial \Omega=\Sigma_{1}-\Sigma_{2}+\sigma$ where $\Sigma_{1}$ and $\Sigma_{2}$ are two oppositely oriented non-intersecting space-like 3-chains and $\sigma$ is the rest of the boundary of $\Omega$. Let $\left\{t, x^{1}, x^{2}, x^{3}\right\}$ be the coordinates of a chart adapted to $\Sigma_{1}$ and $\Sigma_{2}$, i.e. where the images of $\Sigma_{1}$ and $\Sigma_{2}$ are the sets $\left\{p \in \mathcal{M} ; t(p)=t_{1}\right\}$ and $\left\{p \in \mathcal{M} ; t(p)=t_{2}\right\}$ respectively. We require that $j$ has compact support and vanishes on the image of $\sigma$. Thus, equation (94) leads to

$$
\begin{aligned}
\int_{\Omega} d j & =0 \\
& =\int_{\partial \Omega} j \\
& =\int_{\Sigma_{1}} j-\int_{\Sigma_{2}} j+\int_{\sigma} j \\
& =\int_{\Sigma_{1}} j-\int_{\Sigma_{2}} j
\end{aligned}
$$

and so the charges on the surfaces $t=t_{1}$ and $t=t_{2}$ are equal,

$$
Q\left[\Sigma_{1}\right]=Q\left[\Sigma_{2}\right]
$$

i.e. charge is conserved.

Let $V \in \Gamma T \mathcal{M}$ be a future directed time-like normalized vector field. The electric $E_{V}$ and magnetic $B_{V}$ field 1-forms with respect to observers that are integral curves of $V$ are

$$
F=-\tilde{V} \wedge E_{V}-\star\left(\tilde{V} \wedge B_{V}\right)
$$


or

$$
\begin{aligned}
& E_{V}=\iota_{V} F, \\
& B_{V}=-\iota_{V} \star F .
\end{aligned}
$$

Indeed, $\tilde{E}_{\dot{\Gamma}}$ and $\tilde{B}_{\dot{\Gamma}}$ are the electric and magnetic field vectors witnessed by an actual physical observer modelled on an integral curve $\Gamma$ of $V$. A point particle with mass $m$ and charge $q$ is modelled by an observer $C:[0,1] \rightarrow \mathcal{M}$ in the spacetime manifold $\mathcal{M}$ where

$$
\nabla_{\dot{C}} \dot{C}=-\frac{q}{m} \widetilde{\iota_{\dot{C}} F}=-\frac{q}{m} \tilde{E}_{\dot{C}}
$$

The right-hand side of the above expression is the Lorentz force on $C$. Finally, the Poincaré lemma tells us that the second Maxwell equation can be solved on an open subset $\mathcal{U} \subset \mathcal{M}$ to give

$$
F=d A
$$

where $A \in \Gamma \Lambda_{1} \mathcal{U}$ is a Maxwell gauge field 1-form. Therefore, on $\mathcal{U}$ Maxwell's equations simplify to

$$
d \star d A=j
$$

\subsection{Einstein's equations}

Let $\left\{X_{a}\right\}$ be an orthonormal frame for TM. The Ricci 1-forms $P_{a} \in$ $\Gamma \Lambda_{1} \mathcal{M}$ with respect to $\left\{X_{a}\right\}$ are

$$
P_{a}=\iota_{X_{b}} R_{a}^{b}
$$

where $\left\{R_{b}^{a}\right\}$ are the curvature 2 -forms with respect to $\left\{X_{a}\right\}$. Since $\nabla$ is torsion-free the first Bianchi identity (71) is

$$
R_{b}^{a} \wedge e^{b}=0
$$


and so we find that

$$
P_{a} \wedge e^{a}=0
$$

using $R_{a b}=-R_{b a}$ (which follows from (69) and (74)). The Ricci 1forms induce a type $(2,0)$ tensor field Ric $\in \Gamma \mathbf{T}_{2}^{0} \mathcal{M}$ called the Ricci tensor

$$
\text { Ric }=P_{a} \otimes e^{a}
$$

that, using (95), can be shown to be symmetric i.e. $\operatorname{Ric}(X, Y)=$ $\operatorname{Ric}(Y, X)$ for all $X, Y \in \Gamma T \mathcal{M}$. The Einstein 3-forms $G_{a} \in \Gamma \Lambda_{3} \mathcal{M}$ with respect to $\left\{X_{a}\right\}$ are

$$
\begin{aligned}
G_{a} & =R_{b c} \wedge \iota_{X_{a}} \star\left(e^{b} \wedge e^{c}\right) \\
& =R_{b c} \wedge \star\left(e^{b} \wedge e^{c} \wedge e_{a}\right)
\end{aligned}
$$

and induce a type $(2,0)$ symmetric tensor field Ein $\in \Gamma \mathbf{T}_{2}^{0} \mathcal{M}$ called the Einstein tensor

$$
\begin{aligned}
\operatorname{Ein} & =-\frac{1}{2} \star G_{a} \otimes e^{a} \\
& =\operatorname{Ric}-\frac{1}{2} \mathcal{R} g
\end{aligned}
$$

All electromagnetic and matter fields contribute to the stress-energy 3 -forms $\tau_{a} \in \Gamma \Lambda_{3} \mathcal{M}$ which couple to the geometry via the Einstein equations

$$
G_{a}=8 \pi \tau_{a} .
$$

The stress-energy 3 -forms are related to a type $(2,0)$ symmetric tensor field $\mathcal{T} \in \Gamma \mathbf{T}_{2}^{0} \mathcal{M}$ called the stress-energy tensor

$$
\tau_{a}=\star\left[\mathcal{T}\left(X_{a},-\right)\right]
$$

with respect to which (96) can be rewritten

$$
\text { Ein }=8 \pi \mathcal{T} \text {. }
$$




\subsubsection{Conservation laws induced by stress-energy tensors}

Choose the orientation

$$
\star 1=e^{0} \wedge e^{1} \wedge e^{2} \wedge e^{3}
$$

and note that, for example,

$$
\star\left(e^{1} \wedge e^{2} \wedge e^{3}\right)=-e^{0}
$$

because $\left\{e^{0}, e^{1}, e^{2}, e^{3}\right\}$ is orthonormal. Therefore

$$
\begin{aligned}
D \star\left(e^{1} \wedge e^{2} \wedge e^{3}\right) & =-D e^{0} \\
& =-d e^{0}+\omega^{0}{ }_{a} \wedge e^{a} \\
& =0
\end{aligned}
$$

by (65) because $\nabla$ is torsion-free. More generally

$$
D \star\left(e^{a} \wedge e^{b} \wedge e^{c}\right)=0
$$

so using (72)

$$
D G_{a}=D R_{b c} \wedge \star\left(e^{b} \wedge e^{c} \wedge e_{a}\right)+R_{b c} \wedge D \star\left(e^{b} \wedge e^{c} \wedge e_{a}\right)=0 .
$$

Therefore, referring to (96), the covariant exterior derivative of $\tau_{a}$ must vanish :

$$
D \tau_{a}=0
$$

Recall that since $\nabla$ is metric-compatible index "lowering" and "raising" with respect to $\eta_{a b}=g\left(X_{a}, X_{b}\right)$ and $\eta^{a b}=g^{-1}\left(e^{a}, e^{b}\right)$ commutes with $D$.

We already introduced the operator $A_{X}=\nabla_{X}-\mathcal{L}_{X}$ where $X \in \Gamma T \mathcal{M}$.

Note that $A_{X} Y=\nabla_{Y} X, Y \in \Gamma T \mathcal{M}$, since $\nabla$ is torsion-free. If $(\mathcal{M}, g)$ possesses a Killing vector field $K \in \Gamma T \mathcal{M}$, i.e. $K$ satisfies

$$
\mathcal{L}_{K} g=0,
$$


we find that

$$
\begin{aligned}
A_{K}[g(X, Y)] & =\left(A_{K} g\right)(X, Y)+g\left(A_{K} X, Y\right)+g\left(X, A_{K} Y\right) \\
& =g\left(\nabla_{X} K, Y\right)+g\left(X, \nabla_{Y} K\right) \\
& =0
\end{aligned}
$$

since $A_{K}$ annihilates scalar fields and $\nabla$ is metric-compatible. Written in terms of the covariant exterior derivative this reads

$$
\iota_{X_{a}} D K_{b}+\iota_{X_{b}} D K_{a}=0
$$

where $K_{a}=g\left(K, X_{a}\right)$. Thus, introducing $\tau_{K}=K^{a} \tau_{a}=\star[\mathcal{T}(K,-)]$ we find

$$
\begin{aligned}
d \tau_{K} & =D K^{a} \wedge \tau_{a}+K^{a} D \tau_{a} \\
& =D K^{a}\left(X^{b}\right) e_{b} \wedge \tau_{a} \\
& =\frac{1}{2}\left[D K^{a}\left(X^{b}\right)+D K^{b}\left(X^{a}\right)\right] e_{b} \wedge \tau_{a}
\end{aligned}
$$

where the final line follows because $\mathcal{T}$ is symmetric. Using (98)

$$
d \tau_{K}=0
$$

and so $\tau_{K}$, like the electric current 3 -form discussed earlier, is a conserved current.

\subsubsection{Example : Dust}

A relativistic continuum modelled by the stress-energy tensor,

$$
\mathcal{T}^{D}=\rho \tilde{V} \otimes \tilde{V}
$$

where $\rho \in \mathcal{F}(\mathcal{M})$ is the mass-energy density seen by integral observers of the time-like normalized future directed vector field $V$, is called dust. The stress forms are

$$
\tau_{a}^{D}=\rho V_{a} \star \tilde{V}
$$


and so

$$
D \tau_{a}^{D}=D V_{a} \wedge \rho \star \tilde{V}+V_{a} d(\rho \star \tilde{V}) .
$$

Let $\tau_{a}=\tau_{a}^{D}$, i.e. $\tau_{a}^{D}$ is the only contribution to the total stress-energy $\tau_{a}$. Then $D \tau_{a}^{D}=0$ and since $D\left(V^{a} V_{a}\right)=2 V^{a} D V_{a}=0$ we find

$$
\begin{aligned}
& d(\rho \star \tilde{V})=0, \\
& D V_{a} \wedge \star \tilde{V}=0
\end{aligned}
$$

where the latter can be written

$$
\iota_{V} D V^{a}=e^{a}\left(\nabla_{V} V\right)=0
$$

or

$$
\nabla_{V} V=0
$$

i.e. integral curves of $V$ are geodesics on $\mathcal{M}$.

\subsubsection{Common stress forms}

The real-valued scalar field $\varphi \in \mathcal{F}(\mathcal{M})$ with mass $m$ satisfies the Klein-Gordon equation

$$
-d \star d \varphi+m^{2} \varphi \star 1=0
$$

and gives the contribution

$$
\tau_{a}^{K G}=\frac{1}{2}\left(\iota_{X_{a}} d \varphi \wedge \star d \varphi+d \varphi \wedge \iota_{X_{a}} \star d \varphi\right)-\frac{1}{2} m^{2} \varphi^{2} \star e_{a}
$$

to the total stress-energy.

The Maxwell field $F \in \Gamma \Lambda_{2} \mathcal{M}$ satisfies

$$
\begin{aligned}
& d F=0, \\
& d \star F=0
\end{aligned}
$$


in vacuo. Its contribution to the total stress-energy is

$$
\tau_{a}^{E M}=\frac{1}{2}\left(\iota_{X_{a}} F \wedge \star F-F \wedge \iota_{X_{a}} \star F\right) .
$$

It is left as an exercise for the reader to show $D \tau_{a}^{K G}=0$ and $D \tau_{a}^{E M}=0$ when $\tau_{a}=\tau_{a}^{K G}$ and $\tau_{a}=\tau_{a}^{E M}$ respectively, subject to (99), (100) and (101). Hint : Introduce the degree-preserving differential operator $L_{X} \equiv D \iota_{X}+\iota_{X} D$ on frame-valued differential forms and use $L_{X_{a}} e^{b}=0$ to show $L_{X_{a}} \alpha=\nabla_{X_{a}} \alpha$ where $\alpha \in \Gamma \Lambda \mathcal{M}$.

\section{Summary}

The calculus of exterior differential forms is an extremely powerful alternative to conventional vector and tensor calculus. One can view exterior calculus as a "calculus of integrands" since differential forms can be immediately integrated to yield a coordinate-free result. We discussed Lie derivatives and linear connections and demonstrated the power of exterior differential calculus on numerous occasions, most notably by giving an elegant derivation of Bianchi's identities. We briefly discussed how to formulate Newtonian continuum mechanics and General Relativity in a coordinate-free fashion using differential forms and gave some examples.

The amount of literature on the subject of differential geometry is huge and varies quite considerably in depth. We have attempted to summarize the important concepts with applications in mind rather than the underlying mathematical structure. For a comprehensive mathematical account of the foundations of differential geometry see [7] and for a serious introduction to fibre bundle theory see [9]. The other references on differential geometry given in the bibliography are less mathematically demanding. The traditional reference on the topic of differential forms as an alternative to vectors on $\mathbb{R}^{3}$ is [6]. A similar 
reference to [6] is [10]. Comprehensive and accessible introductions to differential geometry are given in [1] and [4]. Applications of exterior calculus to topics in classical mechanics are given in [2]. A comprehensive account of the use of differential forms in spacetime physics is given in [3] from which some of our examples stem. Another good reference containing physical applications is [8]. These notes have been strongly influenced by [3], [5], [2] and [1]. The conventions used here are mostly those in [3].

Exterior differential calculus has many more applications in both physics and mathematics than can be summarized in a single docu-

ment. It is hoped that this article might stimulate its use in novel continuum mechanical studies.

\section{Acknowledgments}

I would like to wholeheartedly thank Robin Tucker for helpful comments and advice on the content of this article.

\section{References}

[1] Abraham, R., Marsden, J.E. \& Ratiu, T. Manifolds, Tensor Analysis and Applications. Springer-Verlag, 1988 (2nd ed.)

[2] Arnold, V.I. Mathematical Methods of Classical Mechanics. Springer-Verlag, 1989 (2nd ed.)

[3] Benn, I.M. and Tucker, R.W. An Introduction to Spinors and Geometry with Applications in Physics. Adam Hilger (IOP publishing Ltd), 1987.

[4] Chern, S.S., Chen, W.H. and Lam, K.S. Lectures on Differential Geometry. World Scientific Publishing, 2000. 
[5] Darling, R.W.R. Differential Forms and Connections. Cambridge University Press, 1994.

[6] Flanders, H. Differential Forms with Applications to the Physical Sciences. Academic, 1963.

[7] Kobayashi, S., Nomizu, K. Foundations of Differential Geometry. Volumes I \& II, Interscience Publishers (John Wiley \& Sons), 1963 \& 1969.

[8] Nakahara, M. Geometry, Topology and Physics. Institute of Physics Publishing, 1990.

[9] Steenrod, N. The Topology of Fibre Bundles. Princeton University Press, 1951.

[10] Weintraub, S.H. Differential Forms - A Complement to Vector Calculus. Academic Press, 1997.

\section{Prvi kurs spoljašnjeg diferencijalnog računa}

UDK 514.753, 514.82, 530.12, 537.6, 532.59

Ovaj uvod u spoljašnje diferencijalne forme na diferencijalnim mnogostrukostima je orijentisan, pre svega, na pedagoški pristup i primenu na konkretne probleme. Teorema Stokes-a, Lie-ov izvod, linearne koneksije sa njihovom krivinom, torzijom i nemetričnošću se diskutuju. Dati su brojni primeri uradjeni ovom metodom i detaljna uporedjenja sa odgovarajućim tradicionalnim vektorskim metodom čine značajan deo ovog rada. Posebno vektorski račun na $\mathbb{R}^{3}$ je izražen pomoću spoljašnjeg računa te se tako tradicionalne teoreme Stokes-a i divergencije zamenjuju snažnijim spoljašnjim izrazom teoreme Stokes-a. 
Primeri iz klasične mehanike kontinuuma kao i fizike prostor-vremena se diskutuju i izvode jezikom spoljašnjih diferencijalnih formi. Brojne prednosti ovog računa u odnosu na tradicionalnu "mašineriju" su naglašene tokom čitavog izlaganja. 